Invited Review Article: Multi-tip scanning tunneling microscopy: Experimental techniques and data analysis

Bert Voigtländer, Vasily Cherepanov, Stefan Korte, Arthur Leis, David Cuma, Sven Just, and Felix Lüpke

Citation: Review of Scientific Instruments 89, 101101 (2018); doi: 10.1063/1.5042346

View online: https://doi.org/10.1063/1.5042346

View Table of Contents: http://aip.scitation.org/toc/rsi/89/10

Published by the American Institute of Physics

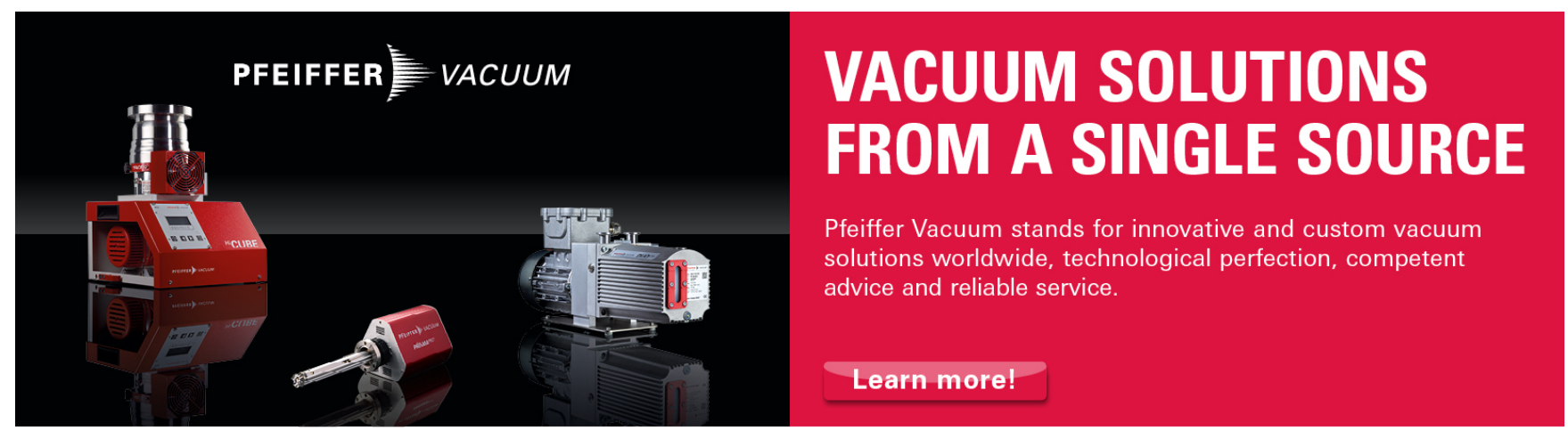




\title{
Invited Review Article: Multi-tip scanning tunneling microscopy: Experimental techniques and data analysis
}

\author{
Bert Voigtländer, a) Vasily Cherepanov, Stefan Korte, Arthur Leis, David Cuma, Sven Just, \\ and Felix Lüpke ${ }^{\text {b) }}$ \\ Peter Grünberg Institut (PGI-3), Forschungszentrum Jülich and JARA-Fundamentals of Future Information \\ Technology, 52425 Jülich, Germany
}

(Received 31 May 2018; accepted 25 August 2018; published online 15 October 2018)

\begin{abstract}
In scanning tunneling microscopy, we witness in recent years a paradigm shift from "just imaging" to detailed spectroscopic measurements at the nanoscale and multi-tip scanning tunneling microscope (STM) is a technique following this trend. It is capable of performing nanoscale charge transport measurements like a "multimeter at the nanoscale." Distance-dependent four-point measurements, the acquisition of nanoscale potential maps at current carrying nanostructures and surfaces, as well as the acquisition of $I-V$ curves of nanoelectronic devices are examples of the capabilities of the multi-tip STM technique. In this review, we focus on two aspects: How to perform the multi-tip STM measurements and how to analyze the acquired data in order to gain insight into nanoscale charge transport processes for a variety of samples. We further discuss specifics of the electronics for multi-tip STM and the properties of tips for multi-tip STM, and present methods for a tip approach to nanostructures on insulating substrates. We introduce methods on how to extract the conductivity/resistivity for mixed 2D/3D systems from four-point measurements, how to measure the conductivity of 2D sheets, and how to introduce scanning tunneling potentiometry measurements with a multi-tip setup. For the example of multi-tip measurements at freestanding vapor liquid solid grown nanowires, we discuss contact resistances as well as the influence of the presence of the probing tips on the four point measurements. Published by AIP Publishing. https://doi.org/10.1063/1.5042346
\end{abstract}

\section{INTRODUCTION}

As microelectronics evolves into nanoelectronics, it is essential to perform electronic transport measurements at the nanoscale. The standard approach is to use lithographic methods for contacting nanostructures, as it is also used in the final nanoelectronic device. However, in research and development stages of nanoelectronic devices other methods of contacting may be more suitable. An alternative approach for the contacting of nanostructures is to use the tips of a multi-tip scanning tunneling microscope, in analogy to the test leads of a multimeter used at the macroscale. The advantages of this approach are (a) in situ contacting of the "as grown" nanostructures still under vacuum allows us to keep delicate nanostructures free from contamination which can be induced by lithography steps performed for contacting. (b) Flexible positioning of the contacting tips and different contact configurations are easy to realize, while lithographic contacts are fixed. (c) Probing with sharp tips can be non-invasive (high ohmic), while lithographic contacts are typically invasive (low ohmic). In order to use a scanning tunneling microscope (STM) for electrical transport measurements at nanostructures, more than one tip is required. This motivates the use of multi-tip scanning tunneling microscopes which give access to the above outlined advantages in nanoprobing. This is in accord with the recent paradigm

\footnotetext{
a) Author to whom correspondence should be addressed: b.voigtlaender@fzjuelich.de

b) Present address: Department of Physics, Carnegie Mellon University, Pittsburgh, PA 15213, USA.
}

shift in scanning probe microscopy which transforms from "just imaging" to detailed spectroscopic measurements at the nanoscale.

Meanwhile, a fast growing community of researchers using multi-tip STM has evolved. Now, it is already hardly possible to compile a comprehensive list of publications in this field. Here, we mention exemplary publications of research groups in this field we are aware of. ${ }^{1-29}$ The community has also established a biannual workshop fostering the scientific exchange. ${ }^{30}$ Moreover, several reviews of the scientific work in this field are available. ${ }^{31-37}$

Researchers operating a single-tip STM often hesitate to extend their experimental capabilities toward multi-tip STM because they are afraid that experimental challenges might multiply with the number of tips. However, modern compact instruments are easy to operate. In our experience, new students only need a couple of months to become acquainted with the multi-tip STM and can subsequently concentrate on scientific topics. Another issue is that a novice in this technique might not be aware of details of the experimental techniques as they are scattered over the literature, or might, as it is nowadays the habit, not be mentioned any more at all.

The aim of this review is to compile the principles of experimental techniques and data analysis methods in one place. The information presented here intends to make it easier for novices in the field to concentrate on scientific topics and benefit from the experience we have gained already.

After the first multi-tip STM was introduced, ${ }^{38}$ several home-built instruments were designed ${ }^{39-46}$ and, today, 
multiple commercial instruments are available as well. ${ }^{47-50}$ In some cases, commercial instruments have been modified. ${ }^{51,52}$ We will not cover the different multi-tip STM designs in this review; however, the general rule that the most compact design results in the most stable operation also applies to multi-tip STM designs. Stability is an important requirement in multi-tip STM since during the electrical measurements, which take some time, the tip positions need to remain fixed. In this review, we will focus on issues which are different in multi-tip STM compared to the operation of a single tip STM.

\section{PRINCIPLE OF A FOUR-POINT MEASUREMENT}

In a two-point resistance measurement, as the one shown in Fig. 1(a), the two current injecting tips are used for voltage probing as well. Therefore, the measured resistance $R_{\text {measure }}$ $=V / I$ includes the intended contribution from the sample resistance. However, it unfavorably also includes the contribution from the two contact resistances as $R_{\text {measure }}=R_{\text {sample }}$ $+2 R_{\mathrm{C}}$ (for simplicity we assume all contact resistances to have the same value). In macroscopic measurements, the contact resistance can often be neglected. However, it should be stressed that the contact resistance is often very large at the STM tip contact to the sample because the contact area is very small so that the four-point measurements are indispensable in resistance measurements with a multi-tip STM. This is even more important for nano-scale objects to be measured because the contacts to these objects are inevitably nano-scale.

In a four-point measurement, the current injecting circuit is separated from the voltage sensing circuit, as shown in Fig. 1(b). If the voltage measurement is performed with a large internal resistance $R_{V} \gg R_{\text {sample }}+2 R_{C}$ (or with a compensation method), the influence of the contact resistances can be neglected. ${ }^{53}$ This is the main advantage of the four-point measurement. The four-point resistance is given by the measured voltage difference divided by the injected current.
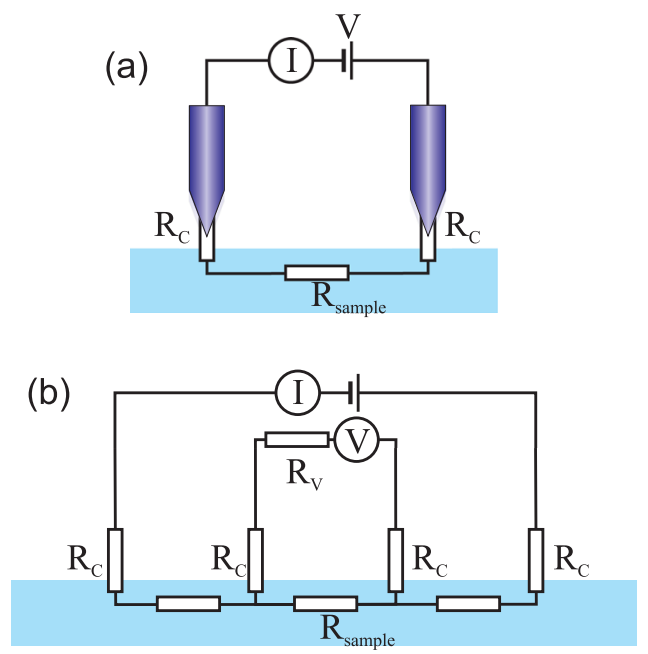

FIG. 1. (a) Two-point resistance measurement in which the contact resistance influences the measured total resistance. (b) Four-point resistance measurement excluding an influence by the contact resistances.

\section{CURRENT MEASUREMENT AND BIAS VOLTAGE}

In single-tip STM, the current measurement and the supply of the bias voltage are usually separated; e.g., the current is measured via the tip contact, while the bias voltage is supplied to the sample or the other way around. In multi-tip STM, this is not possible, as several (different) bias voltages have to be applied and the currents for each tip have to be measured simultaneously. In a multi-tip STM, the sample is usually grounded during a scan, and the current measurement and the supply of a bias voltage are accomplished by using biased current amplifiers as indicated in Fig. 2(a). We consider first the current measurement mode. As shown in more detail in Fig. 2(b), the reference input of the current amplifier (OPA1) is on the bias potential $V_{\text {bias }}$. Additionally, the power supply of the operational amplifier has to be referenced to the bias potential $V_{\text {bias }}$. The output voltage of this transimpedance amplifier (OPA1) is referenced to ground with the difference amplifier (OPA2). ${ }^{44}$

One particular issue in multi-tip STM is the shielding of the wire from the tip to the current amplifier (OPA1). If the guard shield around the tip wire would be grounded, a capacitor

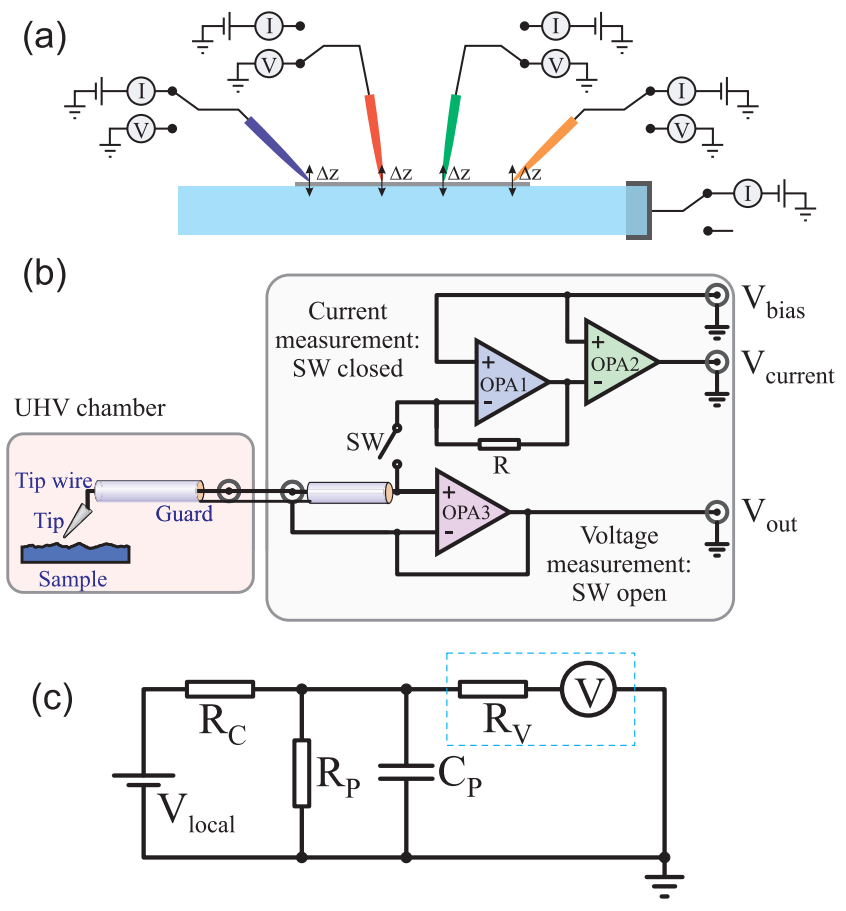

FIG. 2. (a) Principle of the electronics for a multi-tip STM. Each tip can be configured as a biased current probe, e.g., while scanning for STM image acquisition, or as a voltage probe. The shown configuration corresponds to a four-probe resistance measurement of the sample with the outer tips in current measurement mode injecting/draining the current to/from the sample, while the inner tips are in voltage probe mode. The current flowing from the sample to ground can be measured by using an additional (biased) current amplifier or by opening the corresponding switch the sample can be decoupled from ground. (b) A typical electronics consists of a transimpedance amplifier (OPA1) floating on bias voltage $V_{\text {bias }}$ and a difference amplifier (OPA2) referencing the output of the transimpedance amplifier to ground. For the voltage measurement (SW open), a voltage follower (OPA3) is used. In order to prevent capacitive coupling between the signal wire and ground, a guard technique (triax cable) is used. (c) Schematic of the voltage measuring mode including the parasitic components $R_{C}, R_{P}$, and $C_{P}$. The voltage measurement is represented by an ideal voltmeter and an internal resistance $R_{V}$. 
is formed with the grounded shield as one electrode and the tip wire on bias potential as the other capacitor electrode. This parasitic capacitance $C_{P}$ can have values up to about $100 \mathrm{pF}$, which has consequences when (bias) voltage ramps are performed for instance in a four-point measurement. The change of the bias voltage leads to an undesired capacitive current in addition to the current through the sample which is to be measured. This parasitic current can be calculated by $I=C_{P} \cdot d V_{\text {bias }} / d t$. In order to minimize this parasitic capacitive current, the sweeps of the bias voltage can be performed slowly; however, a better approach to minimize the capacitive currents is to reduce the parasitic capacitance between the tip wire and ground. This is done by the well-known concept of guarding, i.e., the shield of the tip wire which is connected to the bias potential $V_{\text {bias. }}$. In this case, during a bias voltage sweep the guard (shield) potential is identical with the tip wire potential. As a result, there is no voltage difference between the two capacitor electrodes such that no capacitive current is induced. Since not all parts of the tip wire are surrounded by the guard, some residual parasitic capacitance between the tip wire and the ground remains. This residual parasitic capacitance to ground $C_{P}$ is about $5 \mathrm{pF}$ in our implementation and can be measured as follows.

The tip is put close to the sample but not yet in tunneling condition; in this case, the capacitive current is the only contribution to the measured current and the capacitance between the tip and grounded sample is included in a realistic way. A bias voltage sweep of triangular shape with a constant voltage change $d V_{\text {bias }} / d t$ leads to a constant current $I_{C}$ which is measured and is used to calculate the residual capacitance between the tip wire and ground, as $C_{P}=I_{C} /\left(d V_{\text {bias }} / d t\right)$.

Another method to check if the capacitive current has an influence on the measured current is to compare the measured currents for bias voltage sweeps of the opposite slope (back and forth pattern of the bias voltage). If no difference in the measured current pattern is found for bias voltage sweeps of opposite directions (i.e., hysteretic behavior), the capacitive current can be neglected (or otherwise averaged out).

The guard can either be connected directly to $V_{\text {bias }}$ or a voltage follower (OPA3) can be used to apply the bias potential to the guard, as shown in Fig. 2(b). The significance of the parasitic capacitance effect depends on the particular measurement conditions. If a large $(\mu \mathrm{A})$ current is injected, the capacitive current can probably be neglected. If, however, a current of the order of nanoampere is injected and the parasitic capacitance is large, the capacitive current can be a substantial fraction of the injected current. Particularly, also if an AC modulation of the bias voltage is applied for spectroscopic measurements, the parasitic capacitive current can be high.

Another issue for the measurement of the current injected to the structure under study is parasitic currents to ground. One parasitic current channel can occur through a finite resistance $R_{P}$ between the wire to the tip and ground. Such a parasitic resistance can result, e.g., from a not perfectly insulating UHV feedthrough. However, this can be measured using the current measurement path of the electronics. For a retracted tip, a vanishing current (pA level at $1 \mathrm{~V}$ applied bias voltage) indicates a sufficiently large resistance. Another type of parasitic resistance is the resistance between the sample and ground. If a current is injected by one tip and drained by a second tip, no parasitic current to ground should be present. This is important in a four-point measurement, as in this case the sample has to be on a floating potential (i.e., not connected to ground). In order to measure the true current through the structure under study, both of the above mentioned kinds of parasitic resistances have to be much larger than the contact resistance between the tip and sample.

\section{VOLTAGE MEASUREMENT}

Up to now, we have considered current measurements; however, in charge transport measurements also the measurement of voltages is required. For instance, for the measurement of the four-point resistance shown schematically in Fig. 2(a), two voltage probes are needed. If the switch (SW) in the electronic scheme in Fig. 2(b) is opened, the electronics is configured as a voltage probe because the operational amplifier OPA3 is wired as a voltage follower. In this case, the STM tip is connected to the input of an operational amplifier with very high input resistance (OPA3); for instance, the specific type of operational amplifier OPA111 has an input resistance of about $R_{V}=10 \mathrm{~T} \Omega$. This voltage follower also acts as an impedance converter and converts the input voltage of very high impedance (in the case of a large tip-sample contact resistance) to a low impedance output signal $V_{\text {out }}$ fed to the subsequent electronics. Due to its very high input resistance, the measurement of the voltage is performed practically without a current to the sample. The measured voltage of the tip corresponds directly to the voltage of the sample at the location contacted by the tip.

In Fig. 2(c), a schematic of the voltage measuring mode with an inner resistance of the voltage measurement (dashed blue rectangle) of $R_{V}=10 \mathrm{~T} \Omega$ is shown together with (possible) parasitic components. The voltage of the sample at the position of the tip $V_{\text {local }}$ is measured properly if the following conditions for the parasitic resistances apply: $R_{V} \gg R_{C}$ and $R_{P} \gg R_{C}$. As $R_{C}$ in tunneling contact may be greater than $10 \mathrm{~T} \Omega$ (specifically on semiconductor samples at zero bias voltage), the voltage sensing tips usually have to be in direct contact to the sample surface to fulfill these requirements.

Another limit is that the contact resistance $R_{C}$ together with the residual parasitic capacitance $C_{P}$ of the signal wire to ground forms a low pass filter and signals beyond the corner frequency are not transmitted (properly). Alternatively explained, the charging of the capacitance $C_{P}$ (current usually on the order of several $\mathrm{pA}$ ) and the corresponding buildup of the voltage at the input of the operational amplifier (OPA) require the usual $R_{C} C_{P}$ time constant. Thus, this time constant has to be smaller than the speed at which the $I-V$ curves are acquired; i.e., the time difference between two voltage measurements has to be longer than the $R_{C} C_{P}$ time constant. Practically, this condition can be confirmed by acquiring the $I-V$ curve for two opposite directions of the current flow. If there is a hysteresis visible, the data acquisition has to be performed slower, or the contact resistance of the voltage probing tip has to be reduced, e.g., by moving the tips closer toward the structure under study. 
If all the previously discussed parasitic effects can be neglected, the following quantities are applied or measured: In the current measurement mode [switch SW in Fig. 2(b) closed] the bias voltage $V_{\text {bias }}$ is applied to the tip via the biased preamplifier. In this case, a current to the sample is injected and simultaneously measured with the tip on bias potential $V_{\text {tip }}=V_{\text {bias }}$. Furthermore, the bias potential is also applied to the guard via the voltage follower OPA3 so that $V_{\text {guard }}=V_{\text {bias }}$. In the voltage probing mode (switch SW open), the local potential on the sample at the position of the tip $V_{\text {local }}$ is measured by the voltage follower OPA3 resulting in $V_{\text {out }}=V_{\text {local }}$. This potential is also applied to the guard: $V_{\text {guard }}=V_{\text {local }}$.

In the following, we briefly introduce a method to measure the sample voltage, if the tip and sample are in tunneling contact. While the electronics scheme described above is the usual way the current and voltage measurement is conducted with a multi-tip STM, we recently proposed an alternative method for the voltage probing using current probes with voltage feedback to measure electric potentials. ${ }^{54}$ One advantage of this technique is the simplified electronics, which uses only four equivalent current measuring units for both current probing and voltage probing, resulting in minimal hardware requirements and corresponding sources of noise. The local sample potential is measured by a software feedback loop which adjusts the corresponding tip bias voltage in such a way that no current flows. The resulting tip voltage is then equivalent to the local sample potential at the tip position. This measurement method allows us to measure the sample potential while the tip is in tunneling contact, thus truly noninvasive four-probe measurements are possible. Details on this approach can be found in Ref. 54. Generally, the noise in any kind of voltage measurement is fundamentally given by the thermal Johnson-Nyquist noise as

$$
V_{\mathrm{J}-\mathrm{N}}=\sqrt{4 k_{B} T \Delta f R_{C}} .
$$

This voltage noise becomes larger for larger contact resistances. For example, for a contact resistance of $R_{C}=1 \mathrm{~T} \Omega$, a bandwidth of $\Delta f=275 \mathrm{~Hz}$, and room temperature, a considerable voltage noise of about $2 \mathrm{mV}$ results. Thus the JohnsonNyquist voltage noise is a limitation for any voltage measurement with a large contact resistance present, i.e., with a voltage probing tip in tunneling contact.

Finally, we would like to mention that also other types of electronics are possible. For example, the current can be injected into the sample from a programmable current source instead of injecting the current by applying a bias voltage to the tip, as described above. In this case, the current source provides a constant current independent of the (fluctuating) contact resistances $R_{C}$. Therefore, it can be argued that a current source is the better option. However, since we measure the current at any time, the present arrangement also provides a complete knowledge of the injected current. Moreover, any tip can be configured flexibly by using software as a current injector or as a voltage probe.

One can also use external measurement equipment like a semiconductor parameter analyzer which provides current sources and the ability to measure voltages. We think, however, that a smooth transition between STM operation and the acquisition of $I-V$ data is advantageous. In this case, both tasks are controlled from the same software and by the same electronics allowing a seamless switching between both. Therefore, we prefer to control everything from the STM software/electronics.

\section{FOUR-POINT MEASUREMENT ON A SURFACE WITH A MULTI-TIP STM}

In this section, we will discuss some practical issues when performing charge transport measurements and take a measurement of the four-point resistance of a $\mathrm{Si}$ sample as an example. The starting situation is that all four tips are placed at their desired positions and all tips are in tunneling contact, while the sample is grounded. In the beginning, we imagined that a measurement sequence would consist of an initial approach of the tips by a certain distance toward the sample surface and then measure the $I-V$ curves. However, it turned out that each tip needs an individual distance of lowering in order to establish a stable tip-sample contact. Therefore, we use the following procedure to perform electrical measurements: The feedback of each tip is stopped and the tip is lowered until a stable current of $0.1 \mu \mathrm{A}-1 \mu \mathrm{A}$ to the sample is established. Afterwards, the tip is not moved until all electric measurements are finished. The z-piezo-motion needed to establish the desired current is about $10-50 \mathrm{~nm}$. As it will be discussed later, this does not mean that the tip indents into the sample by this distance or the tip is plastically deformed by this amount. A small spring constant arises because the tips in multi-tip STM are mounted under an angle of $45^{\circ}$. Due to the relatively small spring constant of the sharp end of the tip of about $50 \mathrm{~N} / \mathrm{m}$, the elastic deformation of the tip exerts a force of about $1 \mu \mathrm{N}$ between the tip and sample, as discussed in more detail in the section. Subsequently, a stable contact is established in the same manner with the other tips. Then the contact of the sample to ground is interrupted, and the electrical measurements are performed.

Figure 3(a) shows depressions left behind after performing the contacting procedure described above. Five indentations from subsequent contacting procedures on a $\mathrm{Si}(111)-(7 \times 7)$ with a diameter of about $40 \mathrm{~nm}$ are imaged. A section through one of the indentations indicated by a white line in Fig. 3(a) is shown in Fig. 3(b). Even this deepest indentation has a depth of only $0.6 \mathrm{~nm}$, which can be compared to an atomic step on the $\mathrm{Si}(111)-(7 \times 7)$ surface of $0.31 \mathrm{~nm}$ present at the right of Fig. 3(b).

For a four-probe resistance measurement as schematically shown in Fig. 2(a), a current has to be injected into and drained from the sample by the outer two tips. We do not supply a defined amount of current using a current source, but apply a voltage to the tips using the electronics described above. A corresponding amount of current flows, which depends on the resistance of the sample and the contact resistance. It may seem as a disadvantage that we cannot directly set the value of the injected current; however, we can measure the current resulting from the applied bias voltage at any time. Even if the contact resistances change slightly during a measurement, this is no problem as the corresponding current is measured. The current is even measured redundantly at the two sourcing and draining tips providing information on possible parasitic current 

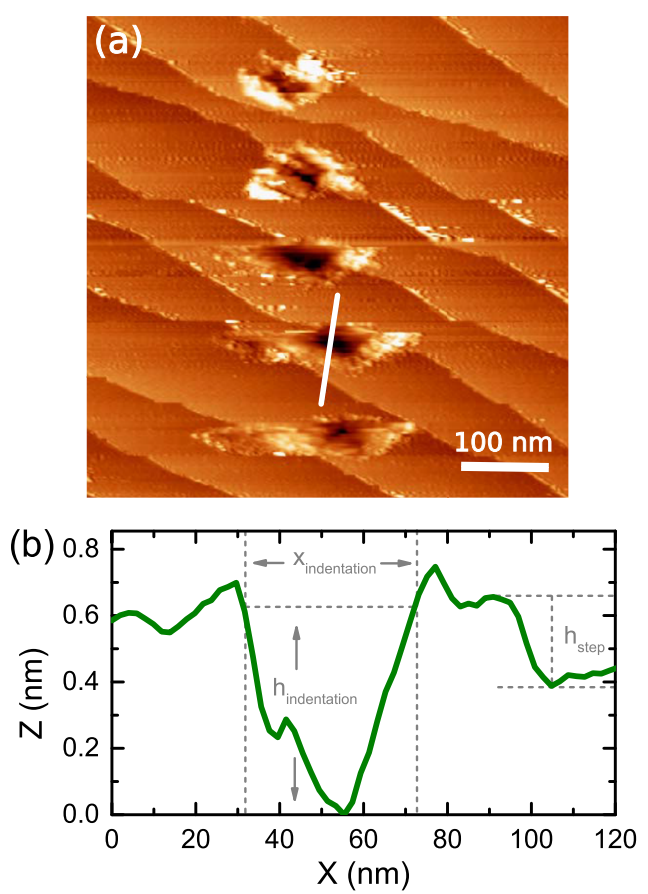

FIG. 3. (a) Indentations left behind after a tip contacting procedures to a $\mathrm{Si}(111)-(7 \times 7)$ surface. (b) Section through one of the indentations [as indicated in (a)] showing a depth of the indentation of $0.6 \mathrm{~nm}$ and a diameter of about $40 \mathrm{~nm}$.

channels. After all, the four-point resistance is independent of the contact resistances and depends only on the flowing current and the voltage difference between the voltage probing tips, which is measured using the voltage probe mode described above. After a complete sweep of the bias voltage, the $I-V$ curve can be plotted from the measured values for current and voltage. In the case of an ohmic conductivity, the $I-V$ curve is linear and the four-point resistance is obtained by a linear fit (around zero current).

Depending on the sample, the resistance to be measured can vary from a very low value on the order of one $\Omega$ to very high values of several $G \Omega$. In the following, we discuss the challenges for the measurement of these very low or very high resistances. For very low resistances, small voltages have to be measured. The voltage measurement can be made more precise by adding an additional operational amplifier (with an amplification of, e.g., 100) behind the voltage follower [OPA3 in Fig. 2(b)]. On the other hand for samples of low resistance, a relatively large current has to flow in order to obtain a reasonable voltage drop. A large current can potentially lead to an undesirable heating of the sample (nanostructure). We have found that a current of $100 \mu \mathrm{A}$ can be tolerated for most samples, but for nanowires of small diameter this may be already a too high current. For samples with a high resistance in the $\mathrm{G} \Omega$ range, the challenge is to inject a sufficient current into the sample. Due to the very sensitive transimpedance amplifiers, currents of less than a nA even approaching the pA range can be measured at a correspondingly low bandwidth. This allows us to measure resistances beyond the $\mathrm{G} \Omega$ range.

While the discussion here concentrates on ohmic and semiconducting samples, the multi-tip STM technique can also be applied to the ballistic transport. ${ }^{14}$ In this case, the multi-tip STM technique has the potential advantage to vary the invasiveness of the voltage probe from almost non-invasive (in the tunneling regime) to an invasive contact regime upon closer tip-sample contact.

\section{EXTRACTING THE RESISTIVITY FROM A FOUR-POINT RESISTANCE MEASUREMENT}

The determined four-point resistance results as the measured potential difference divided by the injected current. However, this quantity is not yet a fundamental property of the sample, as it depends on the positions of the probes on the sample. For the simplest probe configuration, which corresponds to a collinear equidistant arrangement of the probes with a spacing $s$, as shown in Fig. 4, and an infinite (homogenous and isotropic) 3D or 2D sample, simple analytical equations relate the measured four-point resistance $R^{4 p}$ to the resistivity of a 3D sample $\rho_{3 D}$ or the sheet resistivity of a $2 \mathrm{D}$ sample $\rho_{2 D} 35,53,55$

$$
R_{3 D}^{4 p}(s)=\frac{\rho_{3 D}}{2 \pi} \frac{1}{s}
$$

and

$$
R_{2 D}^{4 p}=\frac{\rho_{2 D} \ln (2)}{\pi} .
$$

Here we write the equations in terms of the resistivity $\rho$, while all equations can alternatively be expressed in terms of the conductivity $\sigma=1 / \rho$. Comparing the results of distance-dependent measurements of the four-point resistance to the predictions of Eqs. (2) and (3) allows us to distinguish between a pure $2 \mathrm{D}$ and a pure $3 \mathrm{D}$ system. For a 2D system, the four-point resistance is constant as a function of $s$, while for a 3D system, the four-point resistance obeys a $1 / s$ behavior. Moreover, from the distance-dependent measurements of the four-point resistance the important material parameters $\rho_{3 D}$ or $\rho_{2 D}$ can be determined.

Another advantage of four-point measurements, additionally to its insensitivity on the contact resistances, is that the four-point resistance are largely independent on the size of the current injecting contacts if the tip radius is much smaller than the tip-tip distances. Finite element calculations, which we performed, show that Eqs. (2) and (3) are still valid to a very good approximation, largely independent on the tip diameter.

In the following, we discuss in a brief excursus the units of $\rho_{3 D}$ and $\rho_{2 D}$. If a current flows uniformly through a
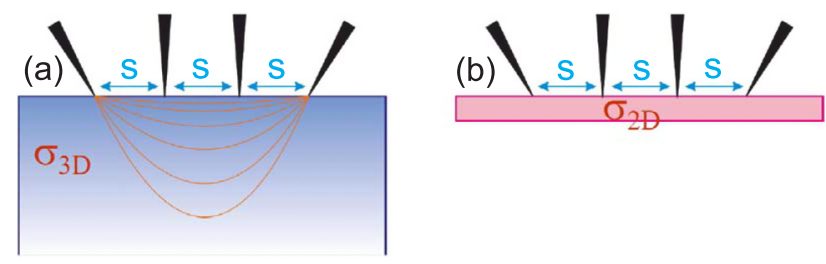

FIG. 4. (a) Four-point resistance measurements on an infinite 3D sample in the collinear equidistant configuration lead to a $1 / s$ distance-dependence. (b) The hallmark of the 2D geometry is a constant four-point resistance. Note that, unlike in the schematic image, the tip spacing $s$ has to be much larger than the thickness of the layer. 


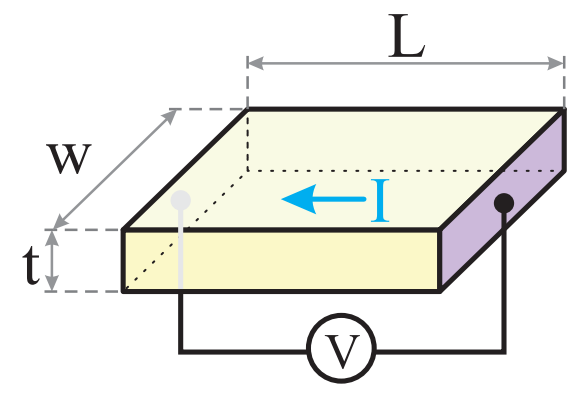

FIG. 5. Schematic of a cuboid through which a current is flowing uniformly.

rectangular resistive cuboid, as shown in Fig. 5, the resistance can be written as

$$
R=\frac{V}{I}=\rho_{3 D} \frac{L}{w t},
$$

with the bulk resistivity $\rho_{3 D}$ having the unit $\Omega \mathrm{m}$. The resistance can be further rewritten as

$$
R=\frac{\rho_{3 D}}{t} \frac{L}{w}=\rho_{2 D} \frac{L}{w},
$$

with the sheet resistivity $\rho_{2 D}=\rho_{3 D} / t$ having the unit $\Omega$. It makes sense to combine $\rho_{3 D}$ with $t$ to one quantity, as both quantities individually may be not (exactly) known, i.e., for thin films. The usual habit to use the unit is $\Omega / \square \equiv \Omega$, pronounced "Ohm per square," for the sheet resistivity, in order to distinguish it from the (measured) resistance $R$. The reason for the term square entering here is, apart from historical reasons, the following: If the rectangular cuboid in Fig. 5 has a square shape, i.e., $L=w$, independent of the size of the square Eq. (5) turns into $R_{2 D}=\rho_{2 D}$.

The expressions Eqs. (2) and (3) relate the measured fourpoint resistance for the $3 \mathrm{D}$ and the $2 \mathrm{D}$ case to the corresponding resistivities $\rho_{3 D}$ and $\rho_{2 D}$, respectively, for the simplest arrangement of the four probes, namely, the collinear equidistant probe configuration. However, there also exist analytical equations for other probe configurations. For the most general configuration of the probes on the sample, as shown in Fig. 6(a), the current is injected and drained by tip 1 and tip 4 , respectively, while the voltage is measured between tip 2 and tip 3. With the tip distance vectors as defined in Fig. 6(a),

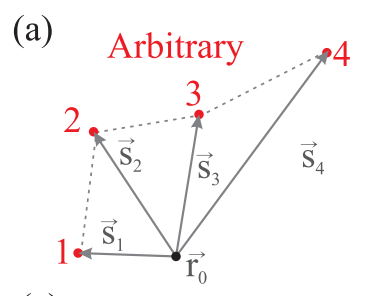

(c)

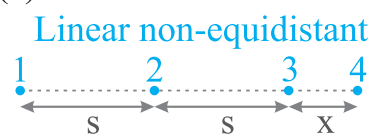

FIG. 6. Tip configurations for a four-probe measurement. (a) Most general non-linear probe arrangement. (b) Linear equidistant probe arrangement. (c) Linear not equidistant probe arrangement with three tips spaced equally by the distance $s$ and one distance $x$ being not equidistant. (d) Square probe arrangement with which an anisotropy of the surface resistivity can be detected. the four-point resistance, based on Ohm's law, for the 3D case results in

$$
\begin{aligned}
R_{3 D}^{4 p}\left(\vec{s}_{1}, \vec{s}_{2}, \vec{s}_{3}, \vec{s}_{4}\right)= & -\frac{\rho_{3 D}}{2 \pi} \cdot\left[\frac{1}{\left|\vec{s}_{3}-\vec{s}_{1}\right|}-\frac{1}{\left|\vec{s}_{3}-\vec{s}_{4}\right|}\right. \\
& \left.-\frac{1}{\left|\vec{s}_{2}-\vec{s}_{1}\right|}+\frac{1}{\left|\vec{s}_{2}-\vec{s}_{4}\right|}\right]
\end{aligned}
$$

and for the $2 \mathrm{D}$ case results in

$$
R_{2 D}^{4 p}\left(\vec{s}_{1}, \vec{s}_{2}, \vec{s}_{3}, \vec{s}_{4}\right)=-\frac{\rho_{2 D}}{2 \pi} \ln \left[\frac{\left|\vec{s}_{3}-\vec{s}_{4}\right| \cdot\left|\vec{s}_{2}-\vec{s}_{1}\right|}{\left|\vec{s}_{3}-\vec{s}_{1}\right| \cdot\left|\vec{s}_{2}-\vec{s}_{4}\right|}\right],
$$

where the vectors $\vec{s}_{i}(i=1,2,3,4)$ start from a common reference point $\vec{r}_{0}$. Note that this equation holds for arbitrary positions of each tip. It is not necessary that the voltage sensing tips are located "in between" the current injecting tips.

The linear non-equidistant probe arrangement, as shown in Fig. 6(c), is experimentally the most efficient way to perform distance-dependent measurements of the four-point resistance, as in this configuration only one of the three distances between the tips, namely, $x$, is changed. In this case, the four-point resistance for the $3 \mathrm{D}$ case results in

$$
R_{3 D}^{4 p}(s, x)=\frac{\rho_{3 D}}{2 \pi} \cdot\left[\frac{1}{x}+\frac{1}{2 s}-\frac{1}{s+x}\right]
$$

and for the $2 \mathrm{D}$ case results in

$$
R_{2 D}^{4 p}(s, x)=\frac{\rho_{2 D}}{2 \pi}\left[\ln \left(\frac{2 s}{x}\right)-\ln \left(\frac{s}{x+s}\right)\right] .
$$

While here the simple qualitative distinction between the 3D case obeying an $1 / s$ dependence and the $2 \mathrm{D}$ case obeying a constant distance dependence from Eqs. (2) and (3), respectively, is lost, there is still a different dependence on the distances $s$ and $x$ between the 2D and 3D cases. So, a distinction between a 2D and a 3D system can again be obtained by distance-dependent measurements of the four-point resistance. . $2,56,57$

In the previous considerations, the contacts were point contacts. In the following, we discuss finite size voltage probes. A finite size voltage probe can be considered as a high conducting (metal) area on a sample of lower conductivity (semiconductor). Without the presence of a probe, a certain potential landscape will establish at the surface of the sample. The presence of a highly conductive voltage probe imposes a constant potential in the area of the contact. Finite element calculations for a $2 \mathrm{D}$ sheet $^{58}$ and our calculations for a surface of a $3 \mathrm{D}$ sample show that this equipotential area leads to a steeper potential gradient in front or behind the probe in the direction of the local potential gradient. However, these calculations show that the potential of the finite size probe corresponds to the potential on the sample at the position of the center of the probe without the probe present. Thus, also a voltage probe of finite size measures the voltage present on the sample at the position of the center of this probe. Therefore, the finite size of the voltage measuring probes is not critical in four-point measurements on 2D and 3D samples and can be neglected. In the case of a 1D sample, the influence of the size of the voltage probing tip can occur for large tip diameters or small 
tip distances. A large tip contact area with the sample provides a shunt path of low resistance for the current and thus the presence of the voltage probes can modify the resistance measurement.

Here, we always considered the case of a (semi-) infinite (3D) $2 \mathrm{D}$ object. In the case of finite systems, correction factors have to be calculated, e.g., for instance for the case of a 3D object of finite thickness on top of a (non-) conducting substrate. Other cases of proximity effects occur for objects of finite lateral size, if the probing tips come close to one of the lateral boundaries of the object, or if the lateral dimension of the object is comparable to the tip spacing $s .{ }^{35,53}$

While for pure $3 \mathrm{D}$ or $2 \mathrm{D}$ systems the situation is simple and clear, already for the still quite simple case of a 2D conducting sheet with $\rho_{2 D}$ on a 3D bulk sample with homogenous conductivity $\rho_{3 D}$ no simple analytic expression for the fourpoint resistance exists. The central question for such a case of multiple conduction channels is: Where does the current flow? The distribution of the current in the different conduction channels determines the potential distribution in the sample and also at the surface, where the potential is measured at two positions in a four-probe measurement. The simplest, often used, model for the current distribution in parallel conduction channels is to consider the different four-point resistances of the individual channels as parallel resistors. While this can be a good approximation in some circumstances, it is unrealistic in other cases. ${ }^{57}$

A more realistic model for the charge transport at a semiconductor surface is to consider a possible 2D surface conductivity $\sigma_{\text {surface }}$, the 3D bulk conductivity $\sigma_{\text {bulk }}$, as well as the depth dependent conductivity in the space charge layer $\sigma_{\mathrm{SC}}$, as schematically shown in Fig. 7(a). The depth dependent conductivity of the space charge layer can be calculated from the surface band bending obtained by solving Poisson's equation. ${ }^{57,59,60}$ In this calculation, the bulk parameters of the semiconductor enter, as well as the position of the Fermi level at the surface with respect to the valence band maximum (surface Fermi level position), which is often pinned to a fixed value independent of the bulk doping due to the high density of states of the surface states. These calculations result in a depth dependent conductivity $\sigma(z)$ which is shown as blue line in Fig. 7(b). This continuous profile $\sigma(z)$ can be approximated by a step-like function to obtain an N-layered structure (green line).
For such an N-layer structure in which a current is injected as shown in Fig. 7(a), the Laplace equation for the potential can be solved..$^{57,61}$ The four-point resistance can be calculated as an integral over Bessel functions with the conductivities of the $\mathrm{N}$ layers as parameters. If the surface conductivity is unknown, its value can be obtained by a fit to experimental distance-dependent four-point measurements. ${ }^{22,57}$ The size of the current injecting probes enters into these calculations as boundary condition but turns out to be not critical. ${ }^{57}$

If the depth dependent conductivity $\sigma(z)$ of the space charge layer is not known, if, e.g., the surface Fermi level position is unknown, the N-layer model can be simplified to a 3-layer model. ${ }^{22}$ In the 3 -layer model, the average conductivity and the average width of the space charge region are only two fit parameters, which can be obtained by a fit to the resistance data. Certainly, this description of the space charge region is now much more approximate, but nevertheless it is possible to obtain an approximate value for the surface conductivity of the studied material from the 3-layer model.

A further point concerning the applicability of the 3layer and N-layer model is that the difference between the two models also depends on the shape of the conductivity profile in the space charge region and the values of the surface and bulk conductivity. If the transport is mostly surface or bulk dominated, then the 3-layer model might be precise enough. But, if the space charge region contributes significantly to current transport in the lateral direction, then the description by the 3-layer model might not be sufficient any more. Also if the space charge region consists of an inversion layer, the approximation by a single step function cannot be very precise and the N-layer model has to be used instead.

A limitation of the approach described above is that the conductivity is calculated straight from the charge carrier densities and effects like transport through p-n junctions (occurring for instance due to inversion layers) are not included properly. More complete treatments of the charge transport in four-probe experiments considering non-equilibrium phenomena described by the drift-diffusion equation are evolving. ${ }^{62}$

\section{Conductivity anisotropy}

Up to now, we have assumed isotropic conductivity, i.e., the conductivity is a scalar quantity. However, this is not always (a)

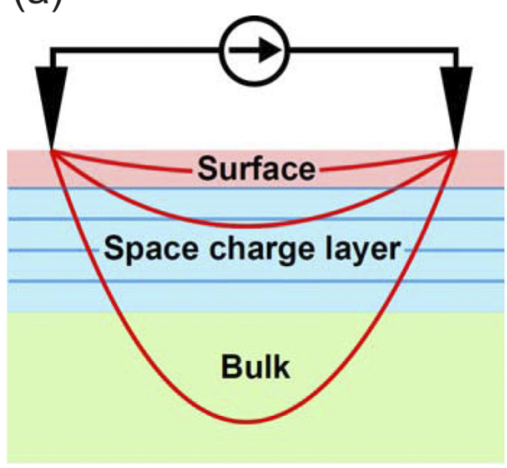

(b)

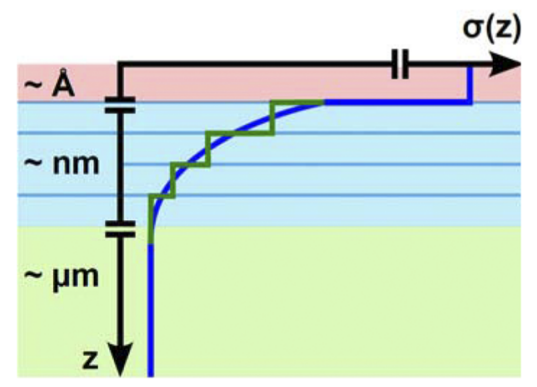

FIG. 7. (a) Schematics of the charge transport channels present at a semiconductor surface. (b) The depth dependent conductivity $\sigma(z)$ of the space charge layer can be calculated by solving Poisson's equation. An N-layer model with $N-2$ layers approximately describing the space charge layer is used to determine the unknown surface conductivity $\sigma_{\text {surface }}$. 
true and the simplest example is a 2D sheet with different conductivities in the $x$ and $y$ direction, $\sigma_{x}$ and $\sigma_{y}$, respectively. An important point is that a linear arrangement of the four probing tips is not suitable to measure the conductance anisotropy. ${ }^{1}$ In order to disentangle an anisotropy in the conductivity, a non-linear configuration of the tips has to be used. A tip arrangement sensitive to an anisotropic surface conductivity is the square arrangement with the tips positioned at the corners of a square as shown in Fig. 6(d). The current injecting tips are positioned at one side of the square and the voltage probing tips at the opposite side. The anisotropic conductivity can be accessed by rotating this square arrangement by an angle $\theta$ in between subsequent fourpoint measurements. ${ }^{1,22,35}$ The four-point resistance resulting from such a measurement configuration can be calculated as $1,22,63$

$$
R(\theta)=C \cdot \ln \left(\frac{\left(\frac{\sigma_{x}}{\sigma_{y}}+1\right)^{2}-4 \cos ^{2} \theta \sin ^{2} \theta\left(\frac{\sigma_{x}}{\sigma_{y}}-1\right)^{2}}{\left(\sin ^{2} \theta+\frac{\sigma_{x}}{\sigma_{y}} \cos ^{2} \theta\right)^{2}}\right),
$$

with $C=1 /\left(4 \pi \sqrt{\sigma_{x} \sigma_{y}}\right)$. Fitting this equation to the result of an angle-dependent measurement determines the components $\sigma_{x}$ and $\sigma_{y}$ of a sample with anisotropic conductivity. The application of the four-point method to anisotropic crystals and surfaces is summarized in Ref. 35. In this reference, it is also shown how the sensitivity for measurements of the anisotropy can be increased by the use of samples of finite size, e.g., circular mesas.

\section{Two-point measurements}

In some cases, it is not possible to apply the more favorable four-point measurements and two-point measurements are performed instead. Apart from the fact that two-point measurements are prone to contact resistances, the measured two-point resistance also depends on the probe contact radius. The following expressions for the two-point resistance for a distance $D$ between the two probes and a probe contact radius $r$ (Fig. 8) can be calculated, ${ }^{64}$ considering the resistance of the contact areas as zero, for the $3 \mathrm{D}$ case as

$$
R_{3 D}^{2 P}=\frac{\rho_{3 D}}{\pi}\left[\frac{1}{r}-\frac{1}{D-r}\right]
$$

and for the $2 \mathrm{D}$ case as

$$
R_{2 D}^{2 P}=\frac{\rho_{2 D}}{\pi} \ln \left[\frac{D-r}{r}\right] .
$$

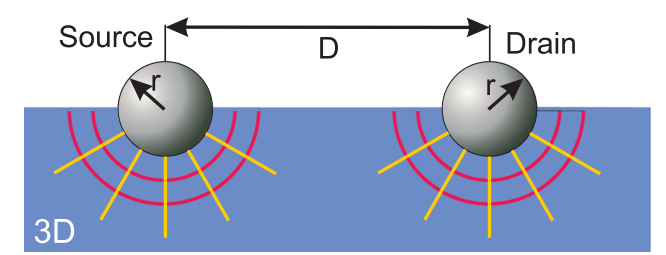

FIG. 8. Schematic of the geometry for a two-point measurement at a 3D sample.

\section{Contact resistance and influence of the tips on the measurement}

In spite of the fact that the four-point measurement is largely independent of the contact resistances it can be helpful to know them. For instance to estimate, if the simpler two-point measurement can be applied instead of the more complicated four-point measurement. We note here that this topic will be considered later in detail for the particular example of contact resistances at nanowires. However, the concepts presented there are applicable in general cases. Another topic is the influence of the presence of the tips on the measured fourpoint resistance, which will also be discussed for the particular example of nanowires later.

\section{TIPS FOR MULTI-TIP STM}

The tips used in multi-tip STM are usually prepared very similar to the ones used in single tip STMs, e.g., often the simple drop-off method is applied. ${ }^{65}$ In multi-tip STM, the tips typically have to be brought together to close distances on the order of a micrometer. In this case, it can happen that also the tip shafts unintentionally come into contact with each other. In order to prevent such a contact at the shaft, the aspect ratio of the tips has to be higher than required for a single tip STM. If we define the ratio of the length from the end of the un-etched shaft of the wire (where it begins to taper) to the tip apex divided by the wire shaft diameter as "aspect ratio" of the tip, then this aspect ratio should be large enough that the shafts of the tips do not touch each other as indicated by the red circle in Fig. 9. In practice (in order to have some safety margin), it has turned out that the aspect ratio of the tips should have a value of at least about two (for four tips azimuthal arranged with a mutual angle of $90^{\circ}$ ). The aspect ratio of the tip can be increased by pulling the tip slowly out of the etching solution during the etching process as described in Ref. 70. We found that alternatively the simple drop-off etching method ${ }^{65}$ can provide tips with a sufficiently high aspect ratio if the wire is immersed somewhat deeper into the etching solution. In order to prepare ultimately sharp tips for multi-tip STM (coated) carbon nanotubes or nanowires have been attached to tungsten tips. ${ }^{66-68}$

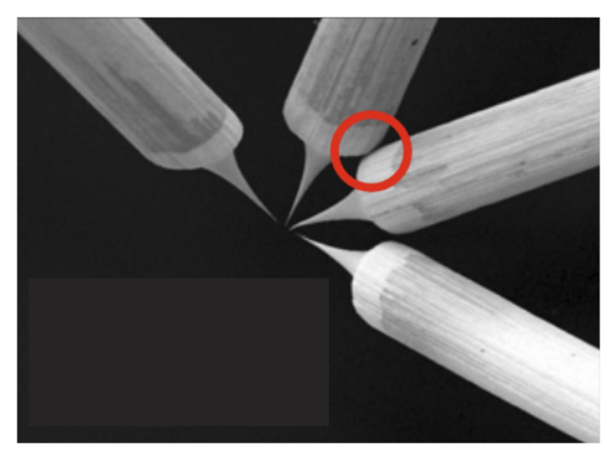

FIG. 9. When the apexes of the tips are brought together, they may contact also at their shafts as indicated by the red circle. Tips with a higher aspect ratio can prevent this problem (diameter of tip wires: $0.2 \mathrm{~mm}$ ). Reproduced with permission from Hobara et al., e-J. Surf. Sci. Nanotechnol. 5, 94 (2007). Copyright 2007 Author(s), licensed under a Creative Commons Attribution 4.0 License. 


\section{Tips directed under an angle toward the surface}

A large difference concerning the tips in single and multitip STM is their angle with respect to the surface plane. While in single tip STM, the axis of the tip is always perpendicular to the sample plane, in multi-tip STM, the angle between the tip and sample is usually $45^{\circ}$. This has several implications for the spring constant of the tip, the resonance frequencies of the tip and the electric contact which establishes between the tip and sample. We will discuss these issues in the following.

Every STM tip is different; however, if prepared with the same etching method, they are quite similar. In order to obtain some quantitative results, we have measured the shape of a typical tungsten STM tip prepared using the drop-off method ${ }^{65}$ by secondary electron microscopy (SEM) imaging. The SEM image of a tungsten tip prepared from a $0.25 \mathrm{~mm}$ polycrystalline tungsten wire, with its characteristic hyperbolic shape, is shown in two magnifications in Fig. 10. This shape of the STM tip was used as an input for finite element calculations. It turns out that the lowest resonance frequencies of the tip are solely determined by the length of the un-etched part of the wire and not by the last etched thin hyperbolically shaped part. This lowest bending resonance frequency of a cylinder clamped rigidly at one side can be calculated according to 69,71

$$
f_{\text {bend }}^{\text {tip }}=\frac{0.14 D}{L^{2}} \sqrt{\frac{E}{\rho}} .
$$

(a)
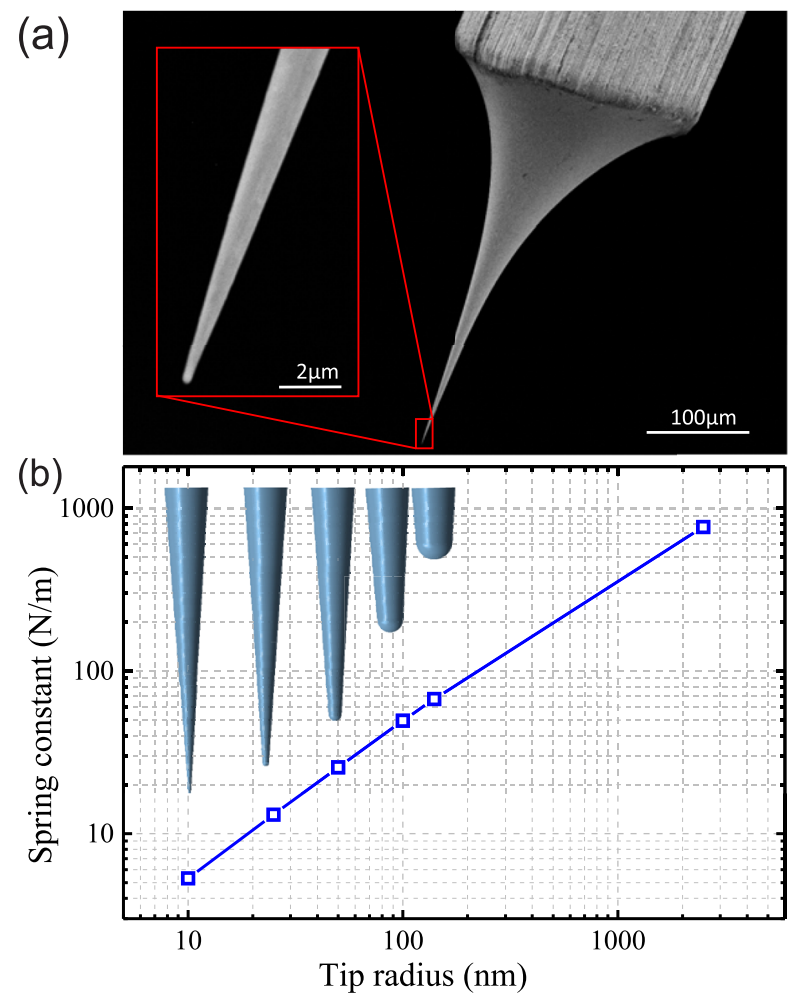

FIG. 10. (a) SEM image of a typical electrochemically etched tip used in multi-tip STM and a zoom to the apex of the tip with a radius of $140 \mathrm{~nm}$. (b) Finite element method (FEM) calculations of the spring constant of tips with different sharpness for a force applied to the hemisphere at the end of the tips under $45^{\circ}$.
For tungsten, Young's modulus is about $E=4 \times 10^{11} \mathrm{~N} / \mathrm{m}^{2}$ and the density is $\rho=1.9 \times 10^{4} \mathrm{~kg} / \mathrm{m}^{3}$. For a tungsten wire of length $L=4 \mathrm{~mm}$ and a diameter of $D=0.25 \mathrm{~mm}$, a resonance frequency of $10 \mathrm{kHz}$ results.

If the bending resonance frequency of the apex part (last several $\mu \mathrm{m}$ ) is calculated using the finite element method (FEM), very high resonance frequencies in the $\mathrm{GHz}$ range result. This result can also be understood from the analytic equation [Eq. (13)]. For a given aspect ratio of a cylinder $(L / D)$, the bending resonance frequency scales as $\propto 1 / L$. Thus, when a cylinder is scaled down in size, the resonance frequency scales as $\propto 1 / L$.

In multi-tip transport experiments, e.g., four-point resistance measurements, currents on the order of $\mu \mathrm{A}$ are typically injected by a tip into the sample. In this regard, the spring constant of the tip is an important quantity which influences the formation of the electrical tip-sample contact. Using the finite element method, the spring constant of a typical tip can be calculated. The spring constant under $45^{\circ}$ for the tip shown in Fig. 10(a) which was approximated at its end by a hemisphere with a radius of $140 \mathrm{~nm}$ was calculated to be $67 \mathrm{~N} / \mathrm{m}$. For comparison, the spring constant of this tip in the vertical direction results in a much higher value of $10000 \mathrm{~N} / \mathrm{m}$. Accordingly, spring constants for sharper tips [tangentially extended from the one shown in Fig. 10(a)] are shown in Fig. 10(b), Here, smaller tip radii, modeled as a hemisphere at the tip end, are used. Also the spring constant of a blunt tip with a tip radius of $2500 \mathrm{~nm}$ was modeled by truncating the tip shape shown in Fig. 10(a). The graph in Fig. 10(b) can be used to estimate the spring constants of similarly shaped tips.

A tip-sample contact is established by moving the tip toward the sample with the corresponding piezo-element. For a hard (tungsten) tip perpendicular to the sample and a soft sample, the tip indents into the sample (elastically or plastically) by the same distance the piezo-element moves. For a hard (e.g., silicon) sample, the tip and sample will be damaged (plastic deformation). On the other hand, the relatively low spring constant for the tip under $45^{\circ}$ leads to an elastic bending of the thinnest part of the tip and prevents thus plastic deformation; i.e., it avoids damaging of the tip and sample to a larger extent than for a perpendicularly oriented tip. In order to illustrate this point a bit more quantitatively, we discuss the results of some contacting experiments in the following.

In these contacting experiments, we used a $\mathrm{Si}(111)$ $(7 \times 7)$ surface on an n-doped sample with a resistivity of $0.01 \Omega \mathrm{cm}$. The tip used was already blunt with a tip radius of about $2.5 \mu \mathrm{m}$, corresponding to a spring constant of about $700 \mathrm{~N} / \mathrm{m}$. After performing different kinds of contacting procedures, the surface is imaged with the same tip afterwards. Here, only one image after all contacting procedures is shown in Fig. 11. Due to the about ten times larger spring constant of this blunt tip compared to fresh tips, the contact forces are also larger by a factor of ten. Despite this, the contact traces on the sample are quite small.

When the tip was lowered by $10 \mathrm{~nm}$ (z-piezo-motion, corresponding to a force of about $7 \mu \mathrm{N}$ ) a current of $25 \mu \mathrm{A}$ flowed at $8 \mathrm{~V}$ bias voltage. The traces left after several of these contacting procedures are only the faint lines with a height smaller 


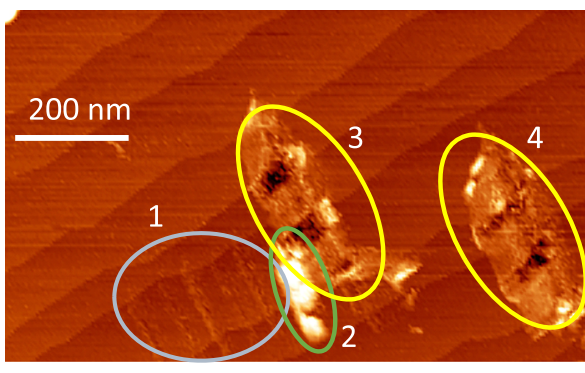

FIG. 11. Traces left on a Si(111) surface after different contacting procedures as described in the text.

than $0.3 \mathrm{~nm}$, highlighted by a gray circle (region 1 ) in the left of Fig. 11. When the tip was lowered by $15 \mathrm{~nm}$, a particularly high current of more than $10 \mathrm{~mA}$ was injected at $8 \mathrm{~V}$ bias voltage. After this contacting procedure no hole, but only additional material on the surface is visible (highlighted in green, region 2). Another tip lowering by $60 \mathrm{~nm}$ resulted in a current of $0.1 \mathrm{~mA}$ at $8 \mathrm{~V}$ bias voltage, and the indentation marks are highlighted in yellow (regions 3 and 4).

While the depth of the indentation holes varies for the different procedures and also for different tips, it can be concluded that the depth of the indentation holes is generally small compared to the change of the tip position by the z-piezo (up to $60 \mathrm{~nm}$ ), even for the case of the exceptionally high spring constant used in this case.

While the tip orientation under $45^{\circ}$ turns out to be an advantage with respect to the damage of the tip and sample during contacting, the relatively low spring constant may lead to the disadvantageous effect of snap-to-contact. ${ }^{69}$ The tip orientated under $45^{\circ}$ acts like an AFM cantilever and attractive van-der-Waals forces can lead to a snap-to-contact. When the tip is stabilized in STM mode at a close distance to the surface, a snap-to-contact will result in strong feedback instabilities switching back and forth between a high (saturation) current when snapped to contact and a vanishing current when the feedback circuit pulls the tip out of contact. The frequency of these oscillatory behaviors depends on the settings of the STM feedback parameters. Fortunately, this effect occurs only very rarely for very long and narrow STM tips oriented under $45^{\circ}$.

\section{CONTACTING CONDUCTING STRUCTURES ON (PARTIALLY) INSULATING SAMPLES}

A conducting substrate can act as a short circuit for the conductance through nanostructures on top. Therefore, the nanostructures of interest often reside on an insulating substrate, such as an oxide layer on a Si wafer. Such a sample structure can also be facilitated to apply a gate voltage. However, a nanostructure on an insulating substrate results in a problem for the approach of the tip to the nanostructure: Without a separate electric contact, the nanostructure is on floating potential such that the usual termination of the automated tip approach used in STM (i.e., stopping further approach upon detection of a tunneling current) does not work.

One way to resolve this problem is to use AFM instead of STM for the approach and find the desired nanostructure on the substrate by AFM scanning. ${ }^{72,73}$ This method is not yet the standard procedure, but very promising. In this section, we introduce several approach methods using an SEM as well as a capacitive approach in order to contact conducting structures on (partially) insulating substrates.

The lateral positioning of the tips of a multi-tip STM toward the structure of interest, while monitoring the tip positions by SEM imaging, is an obvious procedure. The assessment of the vertical tip-sample distance is more difficult. One method is to use the limited depth of focus of an SEM. In this case, the SEM is focused on the sample and the image of the tip becomes sharper the closer the tip approaches toward the sample. Another method to detect the tip-sample distance is SEM imaging with an external detector for the secondary electrons. In this case, a secondary electron shadow of the tip on the sample indicates the tip-sample distance, as shown in Fig. 12. Note that this shadow is not a shadow of the primary electron beam but of the secondary electrons. If the secondary electrons would have to pass the tip from their point of creation on the sample in direct line to the secondary electron detector, they will be blocked by the tip. ${ }^{74}$ This results in a lower secondary electron signal in this line of sight directions, leading to a shadow of the secondary electrons as visible in Fig. 12. As secondary electrons reach the secondary electron detector not only by a line of sight path, this shadow is somewhat diffuse and becomes sharper the closer the tip approaches to the sample.

This secondary electron shadow can be used to monitor the tip approach to a desired structure on the sample down to a distance of several $\mu \mathrm{m}$. In the following three sections, some methods for the final approach bringing the tip into (electrical) contact with the desired structure on the sample are described.

Here we note that SEM and STM imaging can be performed simultaneously on a conducting structure or sample. ${ }^{75}$ For simultaneous STM/SEM imaging, it has to be considered that the SEM electron beam induces an additional current up to the order of $\mathrm{nA}$, which has to be taken into account. Thus, a somewhat higher set-point current has to be selected for STM

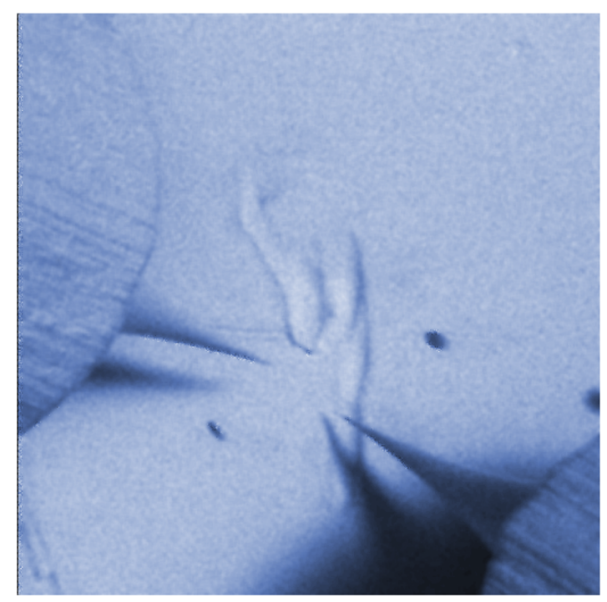

FIG. 12. SEM image of two tips close to a sample. In addition to the tips, two shadows, caused by the blocked secondary electrons are visible on the sample indicating the tip-sample distance (image size: $300 \mu \mathrm{m}$ ). 
imaging such that the additional current from the SEM does not disturb the STM imaging.

\section{Tip approach under SEM control}

The simplest, but also a potentially destructive method, to contact the first tip of a multi-tip STM to a conducting structure on an insulating substrate is to approach the STM tip under SEM control until a slight bending or motion of the tip is visible in SEM images upon the contact to the structure. The other tips can be approached more gently by electrically detecting the contact via the already contacted sample (by the first tip). This method can of course also be used if the desired structure is already contacted by lithographic contacts.

An example in which a $\mathrm{Sb}_{2} \mathrm{Te}_{3}$ nanowire has already been contacted by two gold contacts fabricated using lithographic methods is shown in Fig. 13(a). Such $\mathrm{Sb}_{2} \mathrm{Te}_{3}$ nanowires, with a diameter of about $100 \mathrm{~nm}$, are grown by CVD. ${ }^{76}$ Due to technical limitations, it was only possible to provide two contacts and to perform two-point resistance measurements. Due to this limitation, the quantitative influence of the contact resistance,
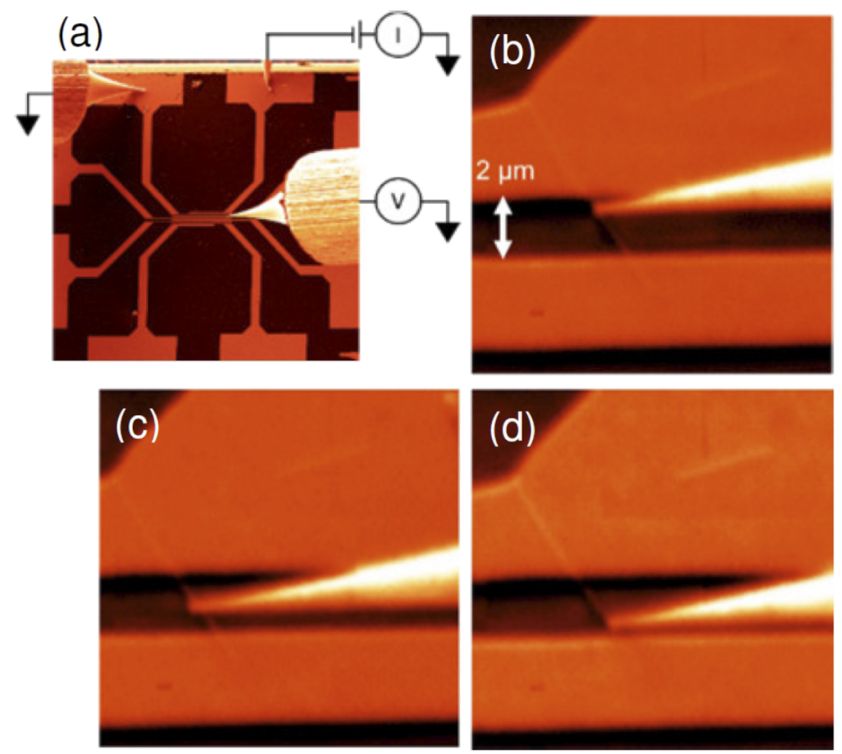

(e)

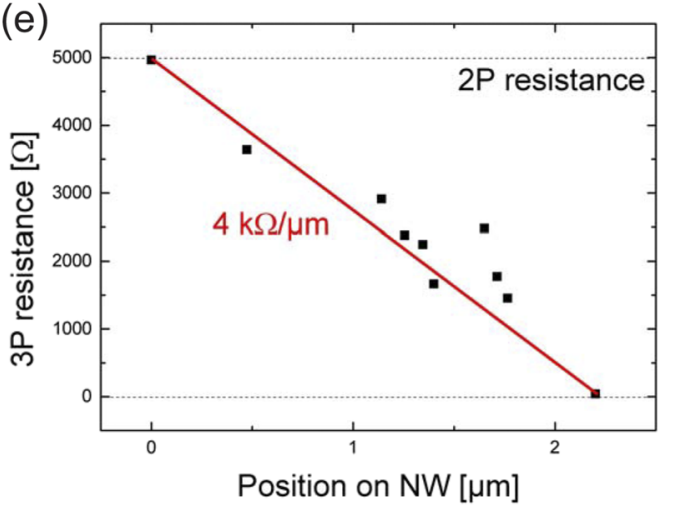

FIG. 13. Topological insulator nanowire with a diameter of $\sim 100 \mathrm{~nm}$ contacted lithographically by gold contacts. Two tips [visible at the very top in (a) ] provide the current injecting contacts to the gold contact pads, while one tip measures the potential along the TI nanowire [(b)-(d)]. The resulting three point resistance is shown in (e) and proves a negligible contact resistance. which is unknown in two-point measurements and adds to the device resistance, remained as an unsolved problem in this two-probe study.

Using the multi-tip STM, a third contact was then provided by one of the tips. The tip was approached under SEM control to the nanowire until a current to one of the lithographic electrodes could be detected. Subsequently, three-point measurements along the nanowire were performed, as depicted in Figs. 13(b)-13(d). The result of this measurement is shown in Fig. 13(e). Since the measured three-point resistance at both ends of the TI nanowire corresponded to the two-point resistance, the contact resistance turns out to be negligible, which however was not clear a priori.

\section{Control of approach via contrast change in the SEM}

The contrast change during SEM imaging can be used to detect the contact of the first tip to an object on an insulating substrate. This method of approach is shown in the following for the example of a graphene flake exfoliated on $\mathrm{SiO}_{2}$. Figure 14(a) shows an SEM image of the graphene flake with the tip positioned above, but not yet in contact to it. The SEM imaging is performed by the detection of secondary electrons as an imaging signal. The images shown in Figs. 14(b) and 14(c) show SEM images after the tip was brought into contact with the graphene flake. In Fig. 14(b), the tip and, thus, also the graphene are biased at $+10 \mathrm{~V}$. The positive potential at the graphene flake attracts secondary electrons and thus leads to less electrons reaching the secondary electron detector, which in turn leads to a darker image of the graphene flake. The opposite occurs if the tip is biased at $-10 \mathrm{~V}$, resulting in a brighter contrast in the SEM image in Fig. 14(c). Thus, SEM imaging with a biased tip is an effective method to detect the point of contact between the tip and graphene flake in a non-destructive way.

\section{Capacitive approach}

If the nanostructure of interest resides on the insulating layer of a back gate, it is possible to approach a tip into a non-invasive contact to the nanostructure by facilitating an AC voltage. For this capacitive approach method (also called AC approach), we consider a sample structure at which the conducting structure to be contacted resides on top of a thin (up to several hundred nanometer thick) insulating layer, e.g., an oxide layer, which then again resides on a conductive substrate.

Such a sample structure is, for example, given by a nanostructure on a silicon-on-insulator (SOI) substrate [as shown in Fig. 15(a)] or a 2D flake on an oxidized silicon wafer. Together with the tip, such a sample structure corresponds to a circuit of capacitors, as shown in Fig. 15(b). For a SOI sample, the lower capacitor $C_{\text {sample-gate }}$ consists of the oxide layer as dielectric and the back-gate silicon as well as the thin sample layer as capacitor plates. $C_{\text {tip-sample }}$ consists of the tip-sample vacuum gap as dielectric, the tip as a top electrode and the thin sample layer as a bottom electrode. Furthermore, there is a capacitance between the tip and the gate electrode $C_{\text {tip-gate }}$.

For the capacitive approach of the tip to the nanostructure at the sample surface, an AC voltage is applied to the back 

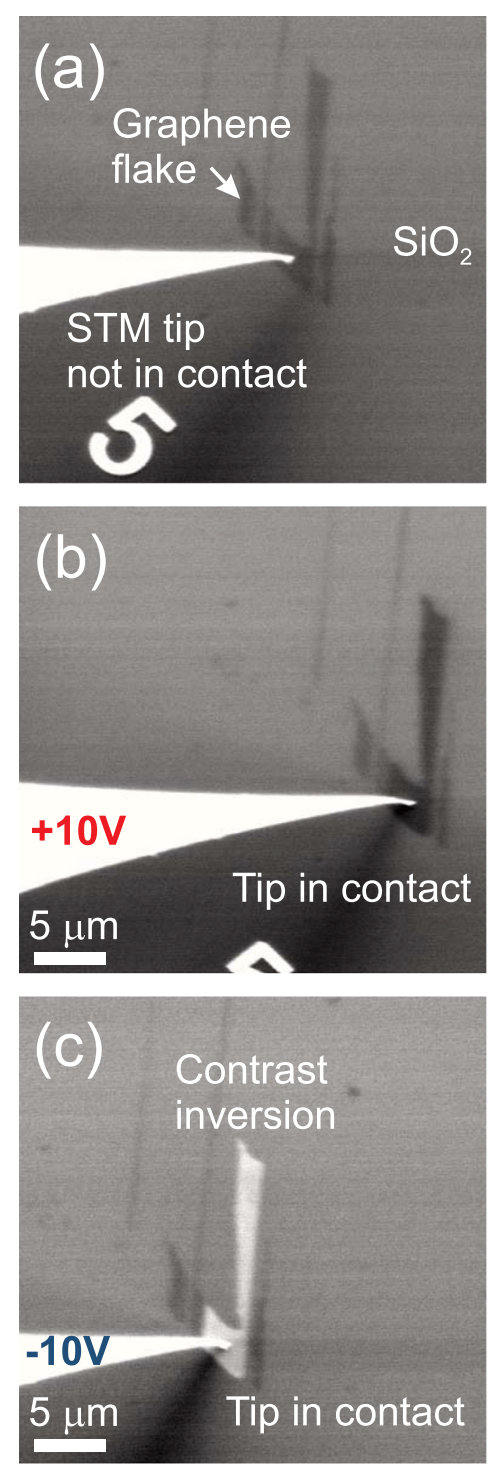

FIG. 14. Contacting of a graphene flake under SEM control using a biased tip. (a) The tip is close to the graphene, but not yet in contact. The images are taken at a beam voltage of $5 \mathrm{kV}$. (b) If the tip comes into contact with the flake and biases it to $+10 \mathrm{~V}$, the secondary electron emission from the graphene becomes lower, leading to an instantaneous darker SEM image. (c) If the tip is biased to $-10 \mathrm{~V}$, the SEM image of the flake switches to brighter upon contact of the tip.

gate of the sample while the tip is held at constant potential. Because the nanostructure is coupled capacitively to the back gate and also to the approaching tip, a capacitive AC current
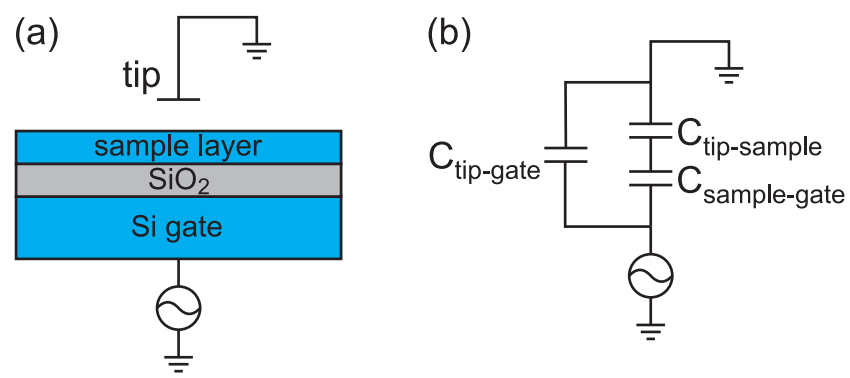

FIG. 15. (a) Silicon-on-insulator (SOI) sample with the top sample layer to be contacted non-invasive by a tip. (b) Equivalent circuit diagram of the different capacitances occurring in this structure. is induced in the tip and can be measured using the regular STM electronics. The applied AC oscillation frequency needs to be chosen such that it is below the bandwidth of the current amplifier (about $1 \mathrm{kHz}$ ) and above that of the STM feedback loop $(\sim 100 \mathrm{~Hz})$; thus, we used an oscillation frequency of $500 \mathrm{~Hz}$. In the following, we briefly consider how this AC current signal is processed by the STM feedback loop.

A typical STM feedback takes the absolute value of the current signal (optionally followed by taking the logarithm). Subsequently, the feedback controller acts like a low pass filter with a bandwidth lower than the frequency of the AC signal applied to the back gate. The resulting DC signal has an amplitude proportional to the capacitive AC signal.

When the tip is still far away from the sample, $C_{\text {tip-sample }}$ and $C_{\text {tip-gate }}$ are small compared to typical values of $C_{\text {sample-gate }}$. Nevertheless, a capacitive current can be measured at the tip. Thus, the current set-point stopping the approach of the tip has to be chosen high enough that the capacitive signal does not stop the approach when the tip is not yet in contact with the sample. In practice, we set the set-point current for the approach (at which the approach is stopped) just above the AC current observed with the tip still far away from the sample. As the tip is approached and comes into tunneling contact with the nanostructure, the capacitance $C_{\text {tip-sample }}$ is effectively bypassed (leading to a large capacitance $C_{\text {tip-sample }}$, as an infinite capacitance can be assigned to two conductors in $\operatorname{contact}^{77}$ ), resulting in a sudden increase in the capacitive current observed at the tip. This increase above the set-point current stops the approach procedure. As a result, the tip is in the tunneling range of the nanostructure and the nanostructure can be contacted by extending the tip. Once the nanostructure is contacted by a tip, its electric potential is fixed and further tips can be approached to it using the usual DC approach methods.

An example in which the capacitive AC approach method was used is a topological insulator layer on a SOI sample allowing gate dependent multi-probe measurements. ${ }^{78}$

\section{CONDUCTIVITY OF 2D SHEETS}

If the sheet conductivity of small 2D flakes from materials such as graphene or transition metal chalcogenides has to be measured, the van der Pauw method is very powerful, as it can be applied to irregularly shaped small flakes. The requirements for the application of the van der Pauw method are a thin 2D sample $(t \ll w, L)$ with a homogenous isotropic conductivity, which is singly connected, i.e., without holes. If the current injecting probes as well as the voltage probes are placed on the periphery of the 2D sample, the sheet resistivity can be obtained from two different four-point measurements, as described in Refs. 79-81.

In Fig. 16, a lithographically structured topological insulator film is shown, which is contacted by four tips in order to perform a van der Pauw measurement. The substrate is a silicon on the insulator (SOI) sample on which the top Si layer was etched away selectively in order to fabricate rectangular silicon mesa structures. Subsequently, a $20 \mathrm{~nm}$ film of the topological insulator material $\left(\mathrm{Bi}_{0.1} \mathrm{Sb}_{0.9}\right)_{2} \mathrm{Te}_{3}$ was deposited by molecular beam epitaxy. ${ }^{82}$ The approach of the tips to the topological 


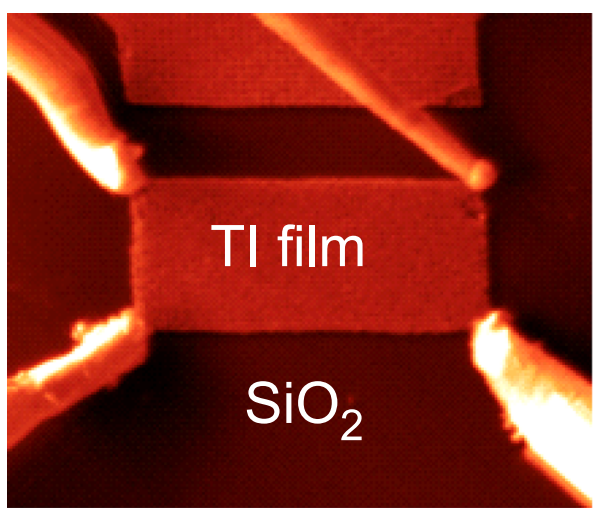

FIG. 16. Van der Pauw measurement on a structured topological insulator film. The SEM image shows a topological insulator film mesa structure $(10 \mu \mathrm{m} \times 4 \mu \mathrm{m})$ contacted with the four tips of the multi-probe instrument.

insulator thin film on top of the non-conducting oxide surface was performed using the AC current upon modulation of the back-gate voltage as discussed in the subsection titled Capacitive approach. Finally, the tips were positioned at the periphery of the topological insulator film and a van der Pauw measurement of the sheet conductance was performed with the four-probe instrument, resulting in a sheet conductivity of $200 \Omega / \square$.

Another method to determine the sheet conductivity of small 2D flakes is to perform four-point measurements with the four tips placed on the flake, in contrast to its periphery as in the case of the van der Pauw method. However, for a small flake the equations valid for an infinite system can no more be applied because the tip spacing $s$ is no more much smaller than the dimension of the flake. In order to relate the measured four-point resistance to a sheet conductivity, finite element calculations can be used. In Fig. 17, the result of finite element (FEM) calculations for a graphene flake, whose shape was measured by SEM, is shown. For a certain position of the current injecting tips at positions 1 and 4, the potential landscape for a homogenous isotropic 2D sheet was calculated

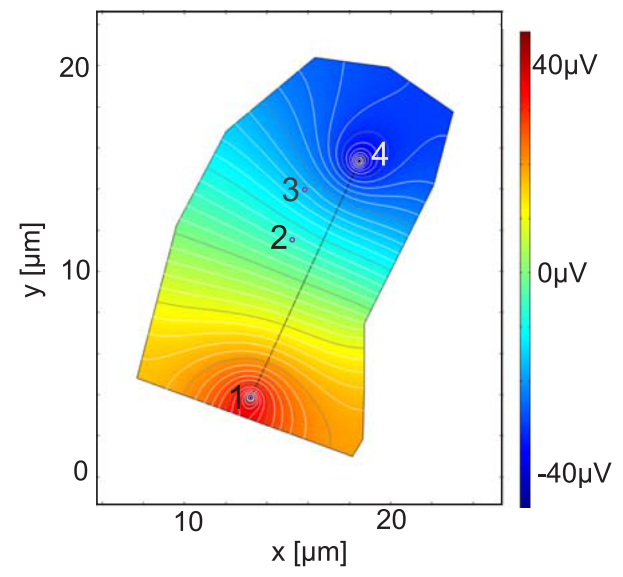

FIG. 17. Calculations of the potential map for a 2D flake with a specific geometry using the finite element method are shown in a color scale. The white and gray lines correspond to equipotential lines with $2 \mu \mathrm{V}$ and $10 \mu \mathrm{V}$ spacing, respectively, for an applied voltage of $1 \mathrm{~V}$ and an assumed sheet conductivity of $1 \mathrm{~S} / \mathrm{m}$. From these calculations, also the voltages relevant for the voltage probing tips 2 and 3 can be obtained. using the FEM method, as shown by the color scale and the equipotential lines. From a comparison between the measured four-point resistance to the one determined from the FEM calculations, the sheet resistivity for a finite small 2D flake is obtained.

\section{SCANNING TUNNELING POTENTIOMETRY (STP)}

Scanning tunneling potentiometry (STP) implemented into a multi-tip scanning tunneling microscope setup allows flexible in situ contacting of samples and simultaneous measurement of the local sample topography and transport field as a result of a lateral current through the sample surface. The resulting spatial and voltage resolution is as low as $\AA$ and $\mu \mathrm{V}$, respectively. Since the invention of STP by Muralt and Pohl, ${ }^{83}$ several different technical implementations of STP have been reported mostly in single-tip STM setups ${ }^{84-86}$ for which the transport field is usually applied to macroscopic sample contacts up to several $\mathrm{mm}$ away from each other. As a result, the current density in the region of measurement can be low, resulting in a bad signal-to-noise ratio. By contrast, a multi-tip STM enables flexible contacting of the sample with several electrodes under ultra-high vacuum conditions. ${ }^{87-89}$

In Fig. 18, a multi-tip STP setup is shown schematically. Two tips inject a lateral current, while a third tip is scanned point-by-point across the sample surface as in regular STM measurements. However, at each scan point after the topography $z(x, y)$ is acquired, the tip is held at constant height for a short time and an additional potential feedback loop is activated to determine the electric potential at that scan point. The final value of the potential feedback is then saved as the sample potential at the momentary position $V(x, y)$. Afterwards, the topography feedback is re-enabled and the scan is continued by moving to the next scan position. In this way, topography and potential maps are simultaneously acquired across the sample surface. More details on this particular STP scheme can be found in Refs. 86 and 88.

A different scheme proposed originally by Muralt and Pohl ${ }^{83}$ is also used. ${ }^{87,89}$ In this implementation, an AC modulation of the tunneling voltage and two feedback loops are used,

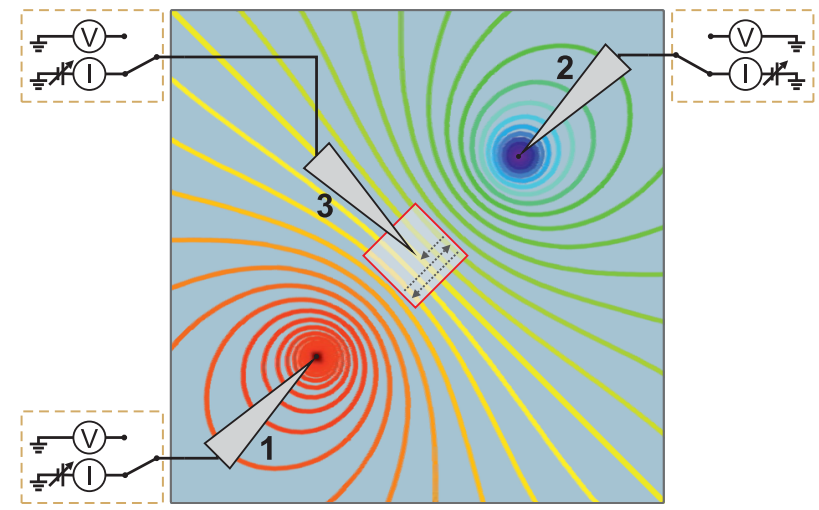

FIG. 18. Schematic of the four-tip STP setup viewed from top. Tips 1 and 2 are in direct contact to the sample surface and inject a lateral current represented by the colored equipotential lines. Tip 3 is in tunneling contact and is scanned across the surface. The scan area is indicated as red square. 
one loop adjusts the distance between the tip and sample using the (rectified) AC component of the tunneling current, the other provides the measurement of the local potential underneath the tip using the DC component. However, it has been shown recently that this implementation is prone to artifacts in the potentiometry signal. ${ }^{89}$ With this AC modulation method, the potentiometry signal is only free from artifacts if the local density of states around zero voltage is constant, leading to a linear $I-V$ characteristic. Any non-linearity in the $I-V$ curve within the range of the AC modulation voltage leads to artifacts due to an asymmetric averaging over the $I-V$ curve when extracting the $\mathrm{DC}$ component by averaging over the $\mathrm{AC}$ signal.

In the following, we demonstrate some principles for STP data analysis, which show how to determine the microscopic transport properties such as the step resistance and the 2D resistance on terraces. Here, we consider as an example the $\operatorname{Si}(111)-(7 \times 7)$ surface.

Figures 19(a)-19(c) show the results of the STP measurements on the $\mathrm{Si}(111)-(7 \times 7)$ surface, where we observe sharp potential drops located at the topographic steps. If charge transport occurs through a $2 \mathrm{D}$ (surface) channel exclusively or the fraction of the current transmitted by the surface channel is known [as it is the case for the $\operatorname{Si}(111)-(7 \times 7)$ surface $^{22,57}$ ], the current density at the position of the potentiometry tip can be calculated from the injected current: ${ }^{10}$ Let the distance between current injecting tip and current draining tip be $d$ and the potentiometry scan is conducted in the middle between these tips at the distance $d / 2$ from both other tips. If a current $I$ is injected into a 2D sheet (and drained in infinity),
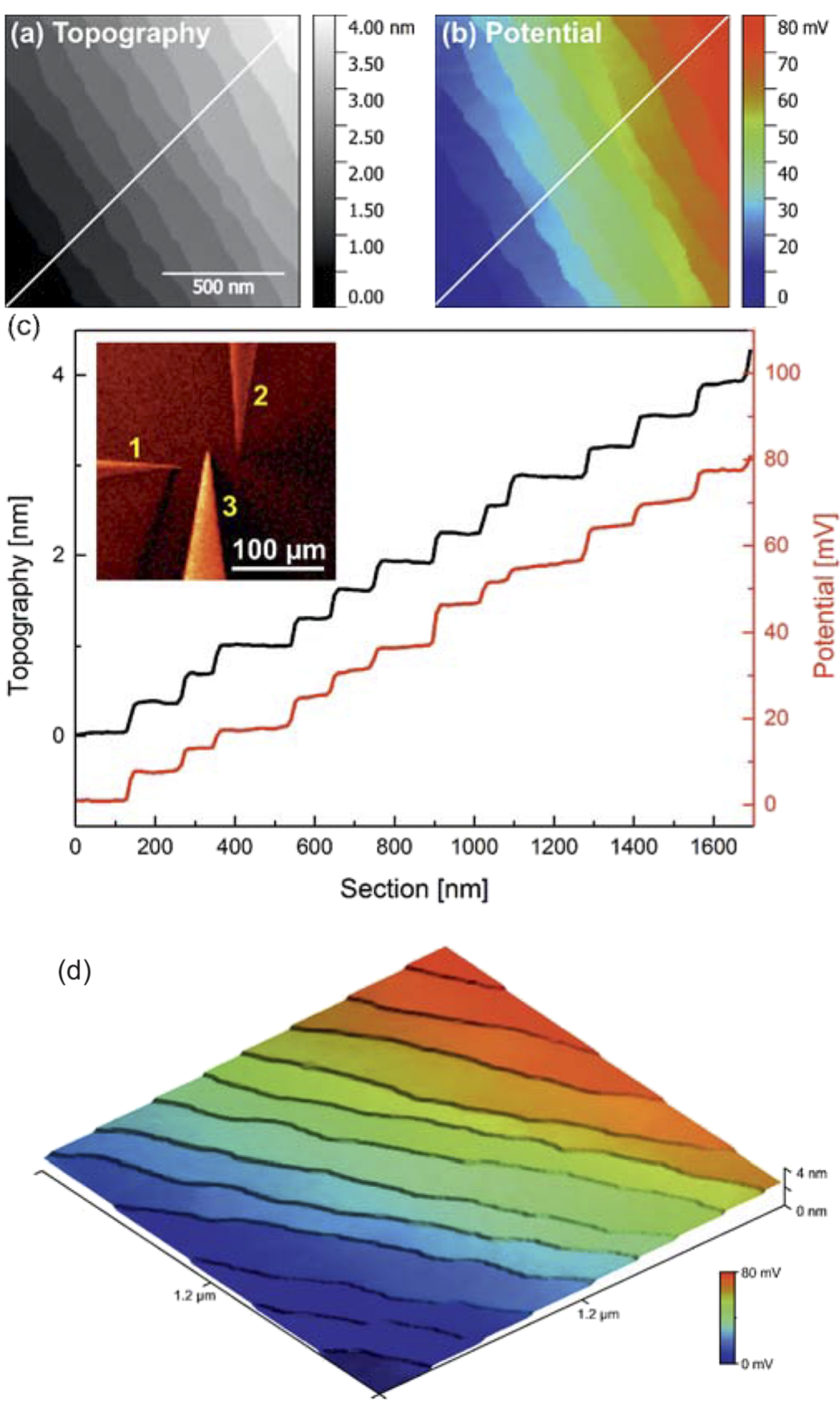

FIG. 19. STP measurements on $\operatorname{Si}(111)(7 \times 7)$. (a) Topography of the surface showing parallel steps with an average terrace width of $130 \mathrm{~nm}$. (b) Corresponding potential map showing sharp jumps in the electric potential. (c) Line profiles indicated in (a) and (b). The inset shows the SEM image of the tip setup on the sample surface. (d) Overlay of the measured topography and potential showing the potential jumps are located at the step edges of the topography. Tunneling conditions are $V=100 \mathrm{mV}$ and $I=0.01 \mathrm{nA}$; the injected lateral current was $8 \mu \mathrm{A}$. 
the current density at a distance $d / 2$ from the point of injection is $j=I /(\pi d)$. As the total current density is a superposition of the current densities from the source and drain, the current density in the middle between the current injecting tips is $j=2 I /(\pi d)$.

Distance-dependent four-probe measurements on the $\operatorname{Si}(111)-(7 \times 7)$ determined that an amount of $70 \%$ of the lateral current is transmitted by the surface conduction channel, in the middle between the current injecting tips with a distance of $d=65 \mu \mathrm{m} .{ }^{22}$ From the injected current density and the respective voltage drops at the step edges and on the terraces, the step resistivity and the terrace resistivity can be determined as $\rho_{\text {step }}=0.101(32) \Omega \mathrm{m}$ and $\rho_{\text {terrace }}=149(14) \mathrm{k} \Omega / \square$, respectively.

Thus, if the local current density at the position where the potentiometry measurements are performed is known, the resistance of the terrace, the steps, as well as also the resistance of defects like domain boundaries can be determined easily. ${ }^{23}$ Moreover in general, scanning tunneling potentiometry allows us to map the potential landscape while a current flows through a nanostructure and has the unique potential to access the spatial distribution of resistances at the nanoscale as can be seen on the overlay of topography with a potential map shown in Fig. 19(d).

An undesired signal in scanning tunneling potentiometry is a thermovoltage between the scanning tip and the sample surface, which arises already from small temperature differences between the tip and sample. ${ }^{86,88}$ This undesired signal can be excluded using an additional potentiometry measurement with the reversed lateral current direction, which inverts the current induced potential signal, but leaves the thermovoltage signal unchanged, or by a measurement without a lateral current applied.

Stable contacts of the current injecting tips to the sample could be established by lowering the respective tips out of tunneling contact until a stable current of about $1 \mu \mathrm{A}$ is observed. The contact stability can be improved by carefully pushing the tips onto the sample surface. To maintain constant contacts over the duration of the measurement, drift, e.g., due to temperature fluctuation of the STM setup, needs to be minimized. In this way, contacts stable for the long measurement durations required in STP (several hours for one scan) can be formed. The resulting current fluctuation over the course of the measurement shown in Fig. 19 is on the order of $1 \%$.

\section{ELECTRICAL MEASUREMENTS AT FREESTANDING NANOWIRES}

Charge transport through nanowires is frequently studied in nanoscience. Particularly, nanowires grown by the vapor liquid solid method (VLS) are of interest. ${ }^{90}$ These nanowires grow in a freestanding manner on the substrate, often perpendicular to the surface, as shown in Fig. 20. The usual way in which the charge transport through these wires is studied is that they are chopped off, put down on an insulating substrate, and subsequently contacted by lithographic methods in order to conduct charge transport measurements. ${ }^{93}$ Direct electrical measurements at the freestanding nanowires are possible with the multi-tip STM technique and have the following
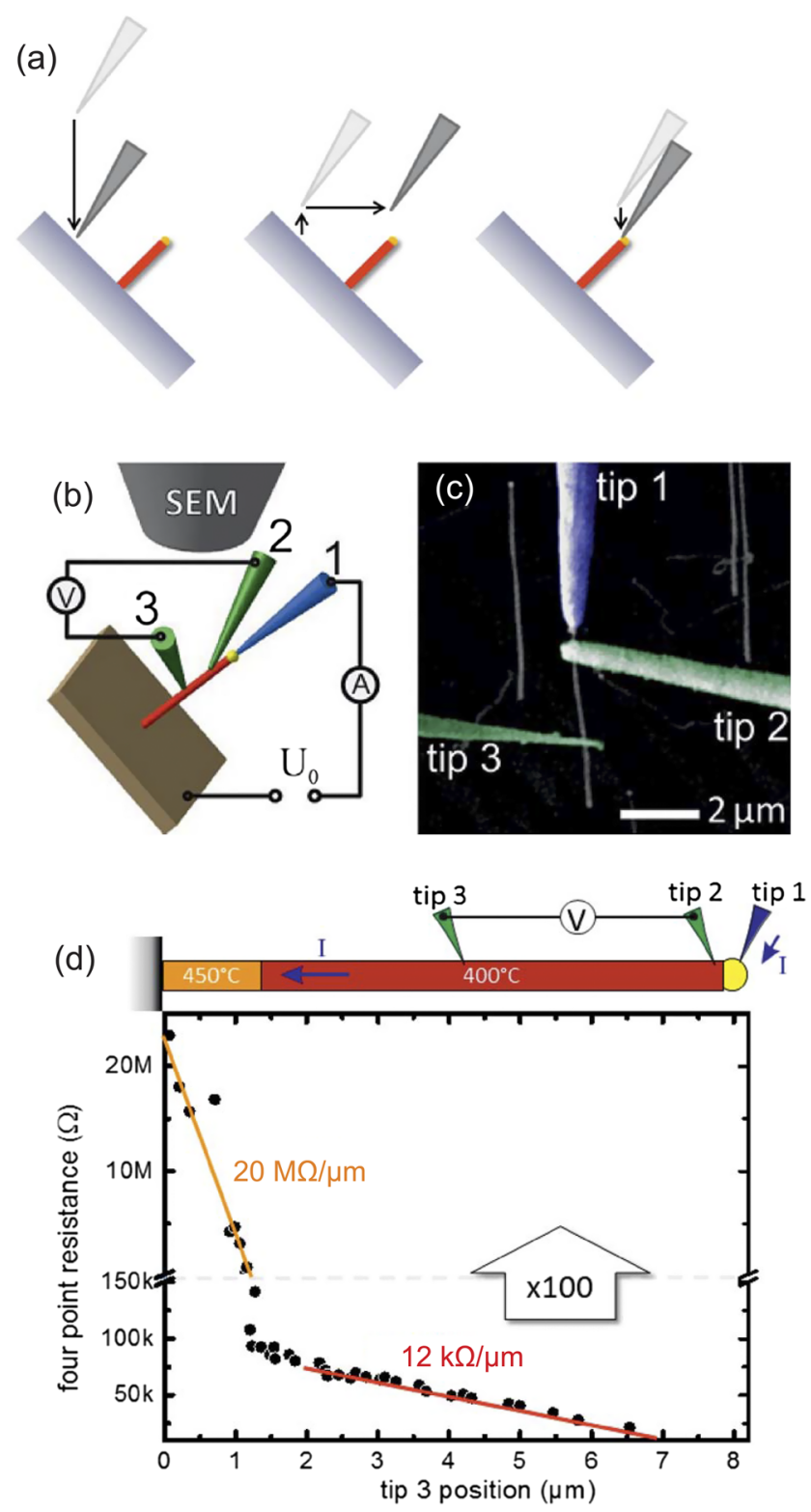

FIG. 20. (a) Schematic for a safe approach process of a tip to the nanowire. (b) Schematic of a four-point measurement on a nanowire with three tips contacting the nanowire. (c) SEM image of a freestanding nanowire contacted by three tips. The STM tips act like the test leads of a multimeter, however, contacting objects like the nanowire at the nanoscale. (d) Resistance profile along a nanowire measured at many different points along the nanowire. On the top in (d) the two step growth process is indicated with the nanowire base grown at high temperature (orange), leading to an undesired high resistance at the nanowire base [as shown in the lower part of (d)]. The upper part of the nanowire (red) has the desired low resistance $(12 \mathrm{k} \Omega / \mu \mathrm{m})$.

advantages: (a) No contamination due to lithographic processing occurs, ${ }^{91,92}$ (b) a resistance/doping profiling along the nanowire is possible with a nm spatial resolution, (c) in situ measurements are possible, avoiding oxidation of the nanowires, ${ }^{94}$ (d) the electrical properties of the transition region between the nanowire and the substrate are accessible.

When monitoring the approach of the tips to the nanowire to be measured using SEM imaging, the usual geometry with the SEM beam perpendicular to the sample is not advantageous. A geometry with a tilted sample allows a better observation of the approach of the tips to the nanowire. Of course 
in this case, the direction perpendicular to the sample appears foreshortened in the SEM images (Fig. 20).

A disadvantage of the tilted sample configuration is that also lateral motions of the tip can lead to an (unwanted) tip sample contact. Since the coarse positioning of the tips is performed by slip stick positioners, a lateral motion of the tips can lead to damaging of the tip (tip crash). One issue which complicates the contacting of the nanowires by the tips is that monitoring this approach by SEM gives good accuracy only in the lateral direction. The depth $(z)$ resolution of an SEM is poor, and thus the information how close the tips have approached toward the sample is very limited. In the following, a safe procedure to bring the tips to a nanowire will be described. In a first step shown in Fig. 20(a), the tip is approached under normal STM feedback control to the substrate sample. The lateral position of the tip is monitored by SEM imaging to be laterally opposite to the desired contact position (upper end of the nanowire in this case), relative to the base of the nanowire. For a sample tilt of $45^{\circ}$, this lateral position on the sample corresponds to the $z$-position of the end of the nanowire as indicated in the left panel of Fig. 20(a). This procedure gives good control on the desired $z$-position along the nanowire. Subsequently, the tip is slightly disengaged from the sample and moved laterally toward the end of the nanowire [the middle panel of Fig. 20(a)]. In a third step, a voltage difference between the tip and sample is applied before the tip is lowered toward the nanowire [the right panel of Fig. 20(a)]. The onset of a current indicates that contact between the tip and the nanowire has been established. This mode should be used to contact the gold particle at the end of the nanowire. A suitable compliance resistance should be used to limit the current because in the worst case a too high current can melt or vaporize the nanowire.

When contacting the side of the nanowire with the voltage probing tips, the voltage probe mode should be used. When the nanowire is contacted, a change in the voltage signal can be observed. Before contact to the nanowire is established, the measured voltage is drifting to the supply voltage limit, in contact it jumps to the nanowire potential at this location.

The example shown in Fig. 20(c) is freestanding GaAs nanowires grown by the VLS method with a diameter of $100 \mathrm{~nm} .{ }^{91,92,94}$ Usually, not much is known about the dopant incorporation and the resulting electrical properties of such freestanding nanowires. The schematics in Fig. 20(b) and an SEM image in Fig. 20(c) show three tips brought into contact with a nanowire, realizing a four-point resistance measurement (with the substrate as fourth contact). Tip 1 injects the current to the nanowire with the substrate acting as current drain, while tip 2 and tip 3 act as voltage probes. In this way, a four-point measurement is realized.

A requirement for a four-point measurement is that the current supplied by the current injecting tips has to be high enough to induce a measurable voltage drop between the voltage probing tips. The demand for the contact resistance of the voltage measurement between the two inner probes [green in Fig. 20(c)] is not stringent due to the high input resistance of the voltage measurement device.

In the above described STM based approach of nanocontacting, four-point measurements can be performed not only in one single configuration, as it is the case for the lithographic approach, but many configurations can be measured by moving the tips along the nanowire (a movie of this procedure can be accessed in the $w_{e} b^{95}$ ). In this way, resistance profiles can be measured along the nanowire with 40 to 50 data points. Figure 20(d) depicts a resistance profile along a GaAs nanowire, which shows a small resistance in the upper part of the wire, while at the nanowire base the resistance becomes very high. ${ }^{91}$ This can be correlated with the two-step growth process of the nanowires: An initial high temperature growth step was used in order to nucleate straight vertically growing nanowires, while a lower growth temperature leads to a more efficient incorporation of the doping species. The identification of the undesired very low doping of the base of the nanowires is a very valuable information triggering subsequent efforts toward improving the electrical properties of the nanowires (increasing the doping) by using different growth conditions.

\section{Contact resistances}

Generally, the four-point measurement method is independent from the contact resistances. While this statement is valid for a wide range of contact resistances, there are limits for the contact resistances. It is obvious that no current can be injected and no voltage can be detected if the respective contact resistances are too large. Therefore it is in some cases desirable to know the contact resistances. In the following, we show how contact resistances of the current injecting contacts as well as the voltage probe contacts can be measured. Here we use the example of VLS grown freestanding nanowires, while the principles are generic and can be applied to other cases as well.

The contact resistance of the current injecting tungsten tip and the gold droplet at the end of the nanowire can be measured as shown schematically in Fig. 21(a), in which a further voltage probing tip is positioned on the nanowire, very close to the upper end. This setup corresponds to a three-point measurement in which the voltage difference $\Delta V$ between the current injecting tip and the voltage probing tip is measured. The corresponding measured voltage as a function of the current injected to the nanowire is shown in Fig. 21(b) and corresponds to a resistance of $130 \mathrm{k} \Omega$. This value represents the resistance of the contact between the tip and the gold droplet at the end of the nanowire, plus the contact resistance between the gold droplet and the nanowire, plus the short nanowire segment between the gold droplet and the voltage probing tip. Since the two last resistance contributions can reasonably be assumed to be small, the measured resistance is an approximation of the contact resistance between the current injecting tip and the nanowire. This resistance was observed to vary only marginally upon repeated contacting of the same nanowire. The data for such a three-point measurement can be acquired at the same time with a normal four-probe measurement and are thus available from a four-point measurement with one voltage probing tip close to the end of the nanowire.

The optimal choice of the polarity of the applied voltage for the three-point measurements depends on the contact resistance at both ends of the nanowire and the nanowire resistance itself. In the current case, the nanowire-gold droplet to 

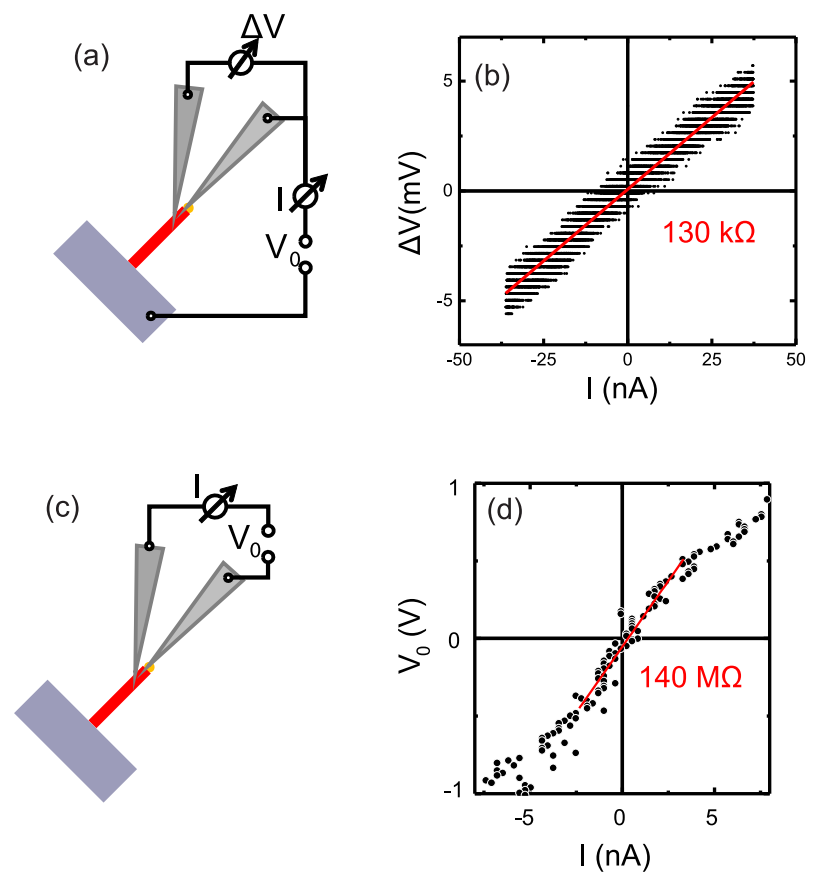

FIG. 21. (a) Schematic of the tip arrangement and the circuit for the measurement of the contact resistance at the current injecting contact at the end of the nanowire. (b) $I-V$ curve for the current injecting contact corresponding to an upper bound of the contact resistance of $130 \mathrm{k} \Omega$. Steps due to the digitization of the voltage measurement are apparent in the graph. (c) Schematic of the tip arrangement and the circuit for the measurement of the contact resistance at the voltage probing contact at the side of the nanowire. (d) $I-V$ curve for the voltage probing contact corresponding to a contact resistance at the voltage probe of $140 \mathrm{M} \Omega$.

tip resistance is low compared to the nanowire resistance and the resistance to the substrate. As a result, the polarity of the current injecting voltage $V_{0}$ should be chosen such that the current injecting tip (not the sample) is on ground. In this case, the voltage at the voltage probing tip is close to zero and can be measured with a higher sensitivity than a small voltage deviation relative to a large (offset) voltage. This case would occur if the sample is chosen to be on ground and the current injecting tip is at the potential $V_{0}$.

Another contact resistance results from the contact between the voltage probing tip and the nanowire. This contact resistance is expected to be larger than the one of the current injecting contact, which is a contact between a metallic tip and the metallic gold droplet. The higher contact resistance to the side of the nanowire is due to an oxide layer and a depletion layer at the surface of the nanowire. This contact resistance can be measured by a two-point measurement between the tip contacting the end of the nanowire and a voltage probe tip to the side of the nanowire, positioned close to the end of the nanowire. A schematic of the measurement setup is shown in Fig. 21(c), and the $I-V$ curve with the resulting two-point resistance of $140 \mathrm{M} \Omega$ is shown in Fig. 21(d). In spite of the fact that this contact is a metal-semiconductor junction, the $I-V$ curve is reasonably linear around zero voltage. Since this resistance is more than thousand times larger than the contact resistance at the nanowire top [Figs. 21(a) and 21 (b)], the measured two-point resistance of $140 \mathrm{M} \Omega$ corresponds to the contact resistance between the voltage probing tip and the nanowire at a position at the side of the nanowire. The value of $140 \mathrm{M} \Omega$ for the contact resistance is still much smaller than the input resistance of the voltage measuring operational amplifier (e.g., $10 \mathrm{~T} \Omega$ ), resulting in a valid voltage measurement.

\section{Influence of the presence of the probing tips}

In the following, we discuss the general question if the four-point resistance determined in a four-point measurement is influenced by the presence of the probing tips. The purpose of the current injecting tips is to source current into the structure under study. Of course the positions of the current injecting/draining tips have to be known and have an influence on the measured four-probe resistance on a particular sample. If there is only one conductance channel present (as in a $2 \mathrm{D}$ conductive sheet), details of the sourcing (e.g., the tip radius) do not influence the measured four-point resistance as the injected current is measured and the equipotential lines are approximately circles, as shown in Fig. 18. The situation is more complicated if the injected current distributes into several conductance channels. ${ }^{11,57}$ Here the distribution of the current among the different conduction channels (e.g., surface state, space charge layer, and bulk) is important. However, often also here the details of the current injection are only a minor issue for the four-point resistance.

Another question, which we will discuss in the following, is, if the presence of the voltage probing tip has an influence on the four-point measurement. It will turn out that conceptually the voltage measured with the voltage probe at a certain position may be different from the voltage at this position without the tip present. We will also discuss how this effect can be measured. In our example of the nanowires, we could not detect an influence of the tips on the four-point resistance.

The voltage measurement at the voltage probing tip performed with a very high internal resistance can conceptually be viewed as a compensation measurement. The potential of the tip is adjusted to a value such that no current flows. This value is the voltage of the sample at the position of the tip. In this case, the Fermi levels of the tip and sample are aligned. Any kind of finite barrier cannot influence the final equilibration and it looks as the presence of the tip (as well as the barrier between the tip and sample) does not influence the voltage measurement.

The influence of the tip on the measured voltage is more indirect, as we will show in the following. The presence of the tip can influence the current flow in the (nano-) structure via a modified band bending at the surface. Since this effect is strongest for semiconductor samples, we will consider these in the following. If the tip comes close to the sample, there is a potential barrier between both, either a vacuum barrier or an oxide barrier. Even if the metal tip comes into direct contact with the semiconductor, a Schottky barrier occurs. In the following, we will consider a vacuum barrier as an example.

The influence of the voltage measuring tip occurs via its influence on the band bending at the surface of the semiconductor (nano) structure through which the current flows. Such a band bending leading to the formation of a space charge layer close to a semiconductor surface occurs due to the 
presence of surface states. ${ }^{60}$ If now a tip is present close to the semiconductor surface, an additional band bending can also be induced by a voltage of the tip relative to the sample. This effect is called tip-induced band bending. ${ }^{96}$ Some of the applied voltage between the tip and sample is dropped within the semiconductor, leading to an additional band bending and the formation/modification of the space charge layer. A simple one-dimensional model of the tip-induced band bending is outlined in the supplementary material, using the Schottky approximation for the space charge layer. However, because the Fermi levels of the tip and sample are aligned at the position of the potential measuring tip, as no voltage is applied, the tipinduced band bending due to a tip-sample voltage difference does not apply.

Another reason for a (additional) band bending at a semiconductor surface in the presence of a tip is the work function difference between the tip metal and the semiconductor [also called contact potential difference (CPD)]. In order to focus on the influence of the work function difference on the formation of a space charge layer, we consider in the following the case that there is no (previous) band bending and no space charge layer due to surface states present: i.e., flat band condition at the surface without the presence of the tip. An initial situation in which the tip and sample are not yet electrically connected is shown in Fig. 22(a). In this situation, the tip and sample share a common vacuum level and the electric field between them vanishes. When the tip and sample are connected electrically and the voltage of the probing tip $V_{0}$ is adjusted such that the current vanishes, as it is done in a four-point measurement, the situation shown in Fig. 22(b) occurs. The alignment of the Fermi levels by the application of the voltage $V_{0}$ induces charge at the tip and the semiconductor surface. The charge at the metal tip is located at the very surface of the tip, while the charge in the semiconductor is distributed over a larger depth, leading to the formation of a space charge layer and correspondingly to a band bending close to the surface of the semiconductor. ${ }^{59,60}$ In this case, the work function difference $\Delta \Phi=\Phi_{\mathrm{M}}-\Phi_{\mathrm{SC}}$ drops partially over the barrier $\Delta \Phi_{\text {barrier }}$ and partially due to the band bending inside the semiconductor $V_{B}$ as $\Delta \Phi=\Delta \Phi_{\text {barrier }}+V_{B}$.

While for a nm sized barrier, the quantities $\Phi_{M}$ and $\Phi_{S C}$ may be different from the corresponding work functions (in the definition of these, an electron has to be removed out of the solid to a large distance compared to atomic distances), the main quantity of importance, the difference $\Delta \Phi$ (local contact potential difference), also exists for an nanometer sized barrier and can be measured using AFM methods. ${ }^{97,98}$

As above considerations show, a contact potential difference between the metal tip and the semiconductor leads to a band bending close to the surface. This local band bending induced by the presence of the tip and due to the contact potential difference can in principle change the charge transport through the current carrying (nano-) structure, as the conductivity can be changed locally due to a modified space charge layer. This modified conductivity can in turn lead to a different voltage at the position of the voltage measuring tips in a four-point measurement.

Up to now, we have considered for simplicity flat band conditions at the semiconductor surface without a tip present.
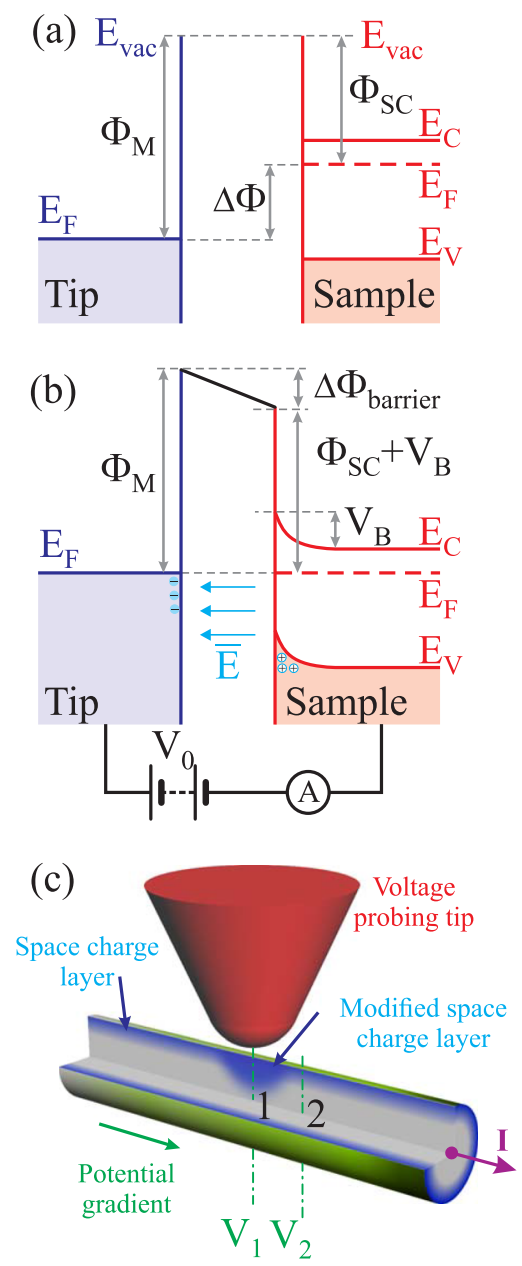

FIG. 22. (a) Schematic of the energy levels of a metal tip and a semiconductor for the case that both are electrically not yet connected (vacuum levels aligned). In order to focus on the work function difference $\Delta \Phi$, no band bending at the free semiconductor surface is assumed (flat band condition). For simplicity, we define $\Phi_{\mathrm{SC}}$ as the difference between the vacuum level and the Fermi level, also if there are no electrons at the Fermi level in a semiconductor. (b) Situation when the tip and semiconductor are connected electrically and the voltage $V_{0}$ has a value leading to a vanishing current $I$ and the Fermi levels of the tip and sample are aligned. In this case, the potential of the voltage measuring tip is that of the semiconductor at this position. The work function difference $\Delta \Phi$ drops partially over the barrier $\Delta \Phi_{\text {barrier }}$ and partially due to the band bending inside the semiconductor $V_{B}$ as $\Delta \Phi=\Delta \Phi_{\text {barrier }}+V_{B}$. (c) The tip-induced band bending leads to a creation or modification of the space charge layer of the nanowire at the position of the tip (position 1), which leads in turn to a modified current in the nanowire.

In most cases, however, there is already a band bending present at the surface of the semiconductor due to the presence of surface states. In this case, the presence of a tip (with a contact potential difference) will modify this initial band bending and will therefore influence the conductivity and in turn the voltage measured by the voltage probing tip.

For the sake of simplicity, we have used a one-dimensional model in Figs. 22(a) and 22(b). For quantitative results, a more realistic three-dimensional treatment has to be performed. ${ }^{96} \mathrm{~A}$ sketch how a modified band bending might look like for the case of a nanowire is shown in Fig. 22(c). The space charge layer present without the influence of the tip is bulged out at the position of the tip (position 1) due to the contact potential difference, which leads in turn to a modified current in the 
nanowire. Due to this modified charge transport, the voltage of the nanowire at the position of the tip changes from $V(1)$ to $V^{\prime}(1)$.

Another small effect may arise due to the tip potential being different from the potential on the semiconductor. This is not the case at position 1 in Fig. 22(c) at which $V_{\text {tip }}=V_{1}$ due to the voltage measurement procedure. At other positions along the semiconductor this is no more true. For example, at position 2 in Fig. 22(c), the potential on the nanowire is $V_{2}$ (potential drop due to the flowing current without tip present), which is different from $V_{\text {tip. }}$. This circumstance can lead to a (slight) change of $V_{2}$ under the influence of the tip potential.

While the above discussion shows that the charge transport may in principle be influenced by the presence of the voltage probing tip, the question of how strong this influence is in a particular case, remains. The main effect is that the modified band bending in the (nano-) structure close to the position of the tip leads to a modified charge transport behavior and in turn to a changed voltage. Thus this effect leads to a modified current through the (nano-) structure. This can be measured as a modified two-point resistance.

The effect should be stronger the more confined the charge transport is, i.e., most pronounced in 1D structures, compared to $2 \mathrm{D}$ and $3 \mathrm{D}$ charge transport. However, in our measurements on nanowires we could not measure such an effect. We did not observe a change in the two-point resistance when moving the tip to or from the nanowire. In another experiment, we changed the voltage of the tip by $\pm 10 \mathrm{~V}$ when the tip was very close to the nanowire. Also in this gating experiment, we could not find a change in the two-point resistance. This means that while conceptually the presence of the voltage probing tip affects the measured voltage, the strength of this effect is often negligible and can be accessed by a measurement of the two-point resistance. A much stronger effect is expected for lithographic contacts, due to their larger size. Thus measuring the four-point resistance with a very local (STM) probe turns out to be an advantage with respect to the influence of the voltage probing contact on the measurement.

\section{Electron beam induced current}

A further technique revealing information about charge transport through nanowires is the measurement of electron beam induced current (EBIC). This measurement is particularly useful in order to detect local doping gradients in nanowires. ${ }^{93}$ In the measurement configuration we present here only one tip is required; however, an SEM is required as well which is often available in multi-tip setups. The principle of an STM based measurement setup to measure electron beam induced current at a freestanding nanowire is shown in Fig. 23(a). An electric circuit is closed by connecting the top of a nanowire with an STM tip and applying (optionally) a voltage $V_{0}$ to the circuit. The signal for the contrast in an SEM image is the (sample) current measured while scanning the SEM beam over the sample. If the high energy electron beam impinges a semiconductor, electron-hole pairs are generated locally. The EBIC image is actually a superposition of a normal sample current image plus the additional current due to the current flowing in the electrical circuit formed when connecting the tip.

If the tip is not yet connected to the nanowire top, the setup corresponds to the sample current imaging mode of

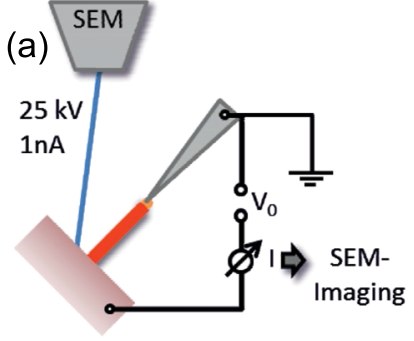

(b)

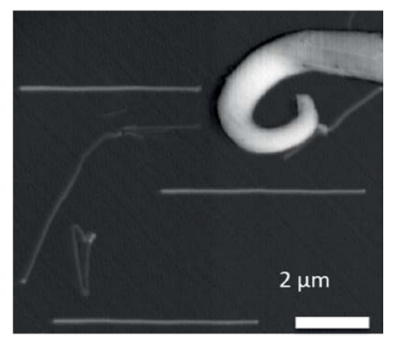

(d)

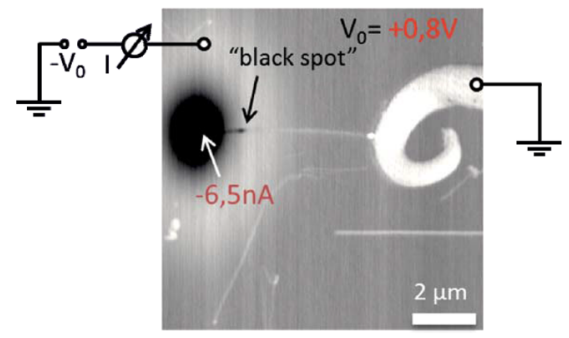

(e)

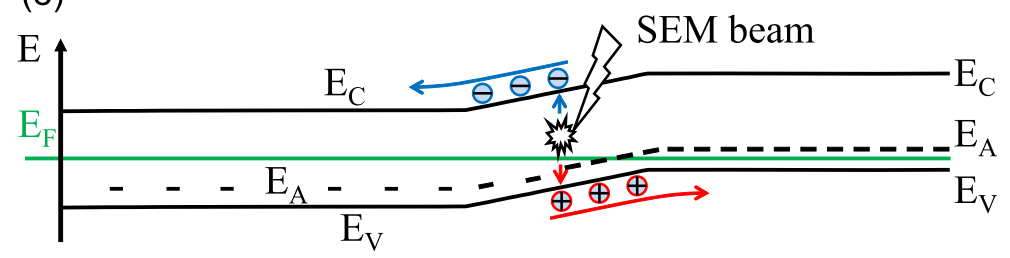

FIG. 23. (a) Schematic for the setup used to acquire electron beam induced current (EBIC) images of freestanding nanowires with an SEM. (b) Sample current image of nanowires together with a (bent) tip not yet contacting a nanowire. (c) EBIC image of a GaAs nanowire which is intrinsic in the lower part and p-doped in the upper part. An EBIC current is detected at the junction between both doping regions. (d) EBIC image of a p-n junction formed by a p-doped nanowire on a n-doped substrate. In this case $V_{0}=-0.8 \mathrm{~V}$. (e) Schematic of the electron-hole generation and the charge separation due to the built in potential at an i-p junction. 
SEM. In this mode, the current flowing from the sample to ground is used as a signal for the SEM image, instead of the usually used signal from an secondary electron detector. A corresponding sample current image showing nanowires and a (bent) tip is shown in Fig. 23(b). An SEM image acquired in the EBIC mode (with $V_{0}=0 \mathrm{~V}$ ) is shown in Fig. 23(c). The GaAs nanowire is undoped in the lower (left) part and pdoped in the upper (right) part of the nanowire. At the position where the undoped and the p-doped parts of the nanowire connect, a strong gradient in the doping profile of the nanowire is present. This junction gives rise to a built-in potential like the one known from a p-n junction, as can be seen in the schematic of a band diagram of an i-p junction shown in Fig. 23(e). When the electron beam hits the junction, electron-hole pairs are generated and separated by the built-in potential. These processes are similar to the ones in a photo-diode when illuminated by light. The separated charges lead to a current which appears as dark spot in the area of the doping gradient. The gradient in the built-in potential separates the electron-hole pair and leads to a current in the direction opposite to the forward current direction of the p-n junction [black spot in Fig. 23(c)] as in the case of a photo-diode. The electron beam induced electron-hole pair generation and charge separation is sketched for an i-p junction in Fig. 23(e). Electrons excited into the conduction band move to the i-doped part, while holes in the valence band move to the $\mathrm{p}$-doped part out of the junction region.

Thus the EBIC technique can be used to quickly detect doping junctions or steep dopant gradients. While the technique was presented here as a qualitative imaging technique, the amount of flowing current can be calibrated in order to perform quantitative current measurements.

Another example for an EBIC signal is a p-doped GaAs nanowire on an n-doped substrate forming a p-n junction at the interface between the nanowire and the substrate [Fig. 23(d)]. In the depletion area (also on the substrate), electron-hole pairs are generated and separated by the built-in potential.

While the EBIC is a particular example, generally the electron beam can be viewed as a further probe, which can be used to inject electrons locally into a (nano-) structure. The spreading of this injected current over the structures on the sample can be detected with the other probes.

\section{OUTLOOK}

In this review, we focused on the most important general experimental techniques and data analysis methods of fourtip STM. Since multi-tip STM is a relatively new technique, some details of the experimental techniques and data analysis methods still have to be developed in order to exploit the power of this method. For instance, in a recent study we measured the four-point resistance of a thin topological insulator film as a function of gate voltage. For the analysis of the data effects like the quantum capacitance of the TI film as well as the contribution of a space charge layer to the conductivity had to be included. ${ }^{57,78}$

In the following, we discuss experimental extensions of the multi-tip STM technique which have either been implemented recently or will be introduced in the near future.
Each of these experimental extensions opens a new sub-field with a lot of potential. In parallel with the implementation of new experimental methods, the corresponding data analysis methods have to be developed as well.

The use of magnetic tips in multi-tip STM opens new possibilities to study the spintronic phenomena, e.g., in topological insulators at the nanoscale. ${ }^{25}$ The possibilities of these studies can still be enhanced by the application of a magnetic field in a multi-tip STM which can be used to revert the magnetization of the tips and investigate the magnetotransport phenomena, such as Hall measurements on the nanoscale.

Another trend is the combination of multi-tip STM with other analysis techniques like angle resolved photoemission spectroscopy (ARPES). In a recent study, we have found that only the UHV transfer between different analysis techniques, in order to study various properties of the same sample, provides the necessary information to interpret certain experimental data successfully. ${ }^{78}$

A further desirable application of multi-tip STM would be the study of the time dependence of transport phenomena on the nanoscale. In order to enable this, the recently developed technique of time-resolved single-tip $\mathrm{STM}^{99}$ has to be transferred to the multi-tip platform.

Also, the usual habit to have (only) four tips in a multitip STM can be expanded to more tips, which can be used for different purposes, for instance, as gate electrodes at nanoelectronic devices.

An important extension of the multi-tip STM technique is the upgrade to AFM operation. If we think about applications in nanoelectronics, most of the samples consist of conducting "target" areas at the surface, separated by non-conducting areas. In order to guide the tip to the conducting areas an AFM operation, instead of or in addition to SEM guided positioning of the tips, can be very useful. ${ }^{6,13}$

The integration of a multi-tip STM in a cryostat with a superconducting magnet would be a big step. With this upgrade to magnetic field operation, the full capabilities of magnetotransport measurements such as Hall effect, weak (anti-) localization, and Shubnikov-de-Haas oscillations will become available at the nanoscale, with the additional flexibility to reposition the probes.

All these experimental extensions will open new exciting research fields and will develop the multi-tip STM technique to an extremely powerful method for the electrical analysis at the nanoscale.

\section{SUPPLEMENTARY MATERIAL}

A simple one-dimensional model of the tip-induced band bending is outlined in the supplementary material.

\section{ACKNOWLEDGMENTS}

The authors would like to thank M. Grob for performing SEM measurements on STM tips and J. Wolters for performing the finite element calculations on the elastic properties of multi-tip STM tips. We would like to thank G. Lu for providing the TI nanowire sample. 
${ }^{1}$ T. Kanagawa, R. Hobara, I. Matsuda, T. Tanikawa, A. Natori, and S. Hasegawa, "Anisotropy in conductance of a quasi-one-dimensional metallic surface state measured by a square micro-four-point probe method," Phys. Rev. Lett. 91, 036805 (2003).

${ }^{2}$ I. Matsuda, M. Ueno, T. Hirahara, R. Hobara, H. Morikawa, Ch. Liu, and S. Hasegawa, "Electrical resistance of a monatomic step on a crystal surface," Phys. Rev. Lett. 93, 236801 (2004).

${ }^{3}$ H. Watanabe, C. Manabe, T. Shigematsu, and M. Shimizu, "Dual-probe scanning tunneling microscope: Measuring a carbon nanotube ring transistor," Appl. Phys. Lett. 78, 2928 (2001).

${ }^{4} \mathrm{O}$. Guise, J. Levy, and J. T. Yates, "Direct measurement of the direction of interface motion in the oxidation of metals and covalent solids $\mathrm{Al}(111)$ and $\mathrm{Si}(100)$ oxidation with $\mathrm{O}_{2}$ at $300 \mathrm{~K}$," Thin Solid Films 496, 426 (2006).

${ }^{5}$ D. K. Lim, O. Kubo, Y. Shingaya, T. Nakayama, Y. H. Kim, J. Y. Lee, M. Aono, H. Lee, D. Lee, and S. Kim, "Low resistivity of Pt silicide nanowires measured using double-scanning-probe tunneling microscope," Appl. Phys. Lett. 92, 203114 (2008).

${ }^{6}$ S. Higuchi, O. Kubo, H. Kuramochi, M. Aono, and T. Nakayama, "A quadruple-scanning-probe force microscope for electrical property measurements of microscopic materials," Nanotechnology 22, 285205 (2011).

${ }^{7}$ J. Homoth, M. Wenderoth, T. Druga, L. Winking, R. G. Ulbrich, C. A. Bobisch, B. Weyers, A. Bannani, E. Zubkov, A. M. Bernhart, M. R. Kaspers, and R. Möller, "Electronic transport on the nanoscale: Ballistic transmission and Ohms law," Nano Lett. 9, 1588 (2009).

${ }^{8}$ S. Bauer and C. A. Bobisch, "Nanoscale electron transport at the surface of a topological insulator," Nat. Commun. 7, 11381 (2016).

${ }^{9}$ M. K. Yakes, D. Gunlycke, J. L. Tedesco, P. M. Campbell, R. L. MyersWard, C. R. Eddy, Jr., D. K. Gaskill, P. E. Sheehan, and A. R. Laracuente, "Conductance anisotropy in epitaxial graphene sheets generated by substrate interactions," Nano Lett. 10, 1559 (2010).

${ }^{10}$ S.-H. Ji, J. B. Hannon, R. M. Tromp, V. Perebeinos, J. Tersoff, and F. M. Ross, "Atomic-scale transport in epitaxial graphene," Nat. Mater. 11, 114 (2012).

${ }^{11}$ C. M. Polley, W. R. Clarke, J. A. Miwa, M. Y. Simmons, and J. W. Wells, "Microscopic four-point-probe resistivity measurements of shallow, high density doping layers in silicon,” Appl. Phys. Lett. 101, 262105 (2012).

${ }^{12}$ C. M. Polley, W. R. Clarke, J. A. Miwa, G. Scappucci, J. W. Wells, D. L. Jaeger, M. R. Bischof, R. F. Reidy, B. P. Gorman, and M. Simmons, "Exploring the limits of n-type ultra-shallow junction formation," ACS Nano 7, 5499 (2013).

${ }^{13}$ M. Hirose, E. Tsunemi, K. Kobayashi, and H. Yamada, "Influence of grain boundary on electrical properties of organic crystalline grains investigated by dual-probe atomic force microscopy," Appl. Phys. Lett. 103, 173109 (2013).

${ }^{14}$ J. Baringhaus, M. Ruan, F. Edler, A. Tejeda, M. Sicot, A. Taleb-Ibrahimi, A. P. Li, Z. Jiang, E. H. Conrad, C. Berger, C. Tegenkamp, and W. A. de Heer, "Exceptional ballistic transport in epitaxial graphene nanoribbons," Nature 506, 349 (2014).

${ }^{15}$ L. Barreto, L. Kühnemund, F. Edler, C. Tegenkamp, J. Mi, M. Bremholm, B. B. Iversen, C. Frydendahl, M. Bianchi, and P. Hofmann, "Surface-dominated transport on a bulk topological insulator," Nano Lett. 14, 3755 (2014).

${ }^{16}$ B. V. C. Martins, M. Smeu, L. Livadaru, H. Guo, and R. A. Wolkow, "Conductivity of $\mathrm{Si}(111)-(7 \times 7)$ : The role of a single atomic step," Phys. Rev. Lett. 112, 246802 (2014).

${ }^{17}$ M. Wojtaszek, J. Lis, R. Zuzak, B. Such, and M. Szymonski, "Inversion layer on the $\mathrm{Ge}(001)$ surface from the four-probe conductance measurements," Appl. Phys. Lett. 105, 042111 (2014).

${ }^{18}$ M. Kolmer, P. Olszowski, R. Zuzak, S. Godlewski, C. Joachim, and M. Szymonski, "Two-probe STM experiments at the atomic level," J. Phys.: Condens. Matter 29, 444004 (2017).

${ }^{19}$ C. Durand, P. Capiod, M. Berthe, J. P. Nys, C. Krzeminski, D. Stievenard, C. Delerue, and B. Grandidier, "Nanoscale carrier multiplication mapping in a Si diode," Nano Lett. 14, 5636 (2014).

${ }^{20}$ A. M. Lord, T. G. Maffeis, O. Kryvchenkova, R. J. Cobley, K. Kalna, D. M. Kepaptsoglou, Q. M. Ramasse, A. S. Walton, M. B. Ward, J. Köble, and S. P. Wilks, "Controlling the electrical transport properties of nanocontacts to nanowires," Nano Lett. 15, 4248 (2015).

${ }^{21}$ A. M. Lord, J. E. Evans, C. J. Barnett, M. W. Allen, A. R. Barron, and S. P. Wilks, "Surface sensitivity of four-probe STM resistivity measurements of bulk ZnO correlated to XPS," J. Phys.: Condens. Matter 29, 384001 (2017).
${ }^{22}$ S. Just, M. Blab, S. Korte, V. Cherepanov, H. Soltner, and B. Voigtländer, "Surface and step conductivities on Si(111) surfaces," Phys. Rev. Lett. 115, 066801 (2015).

${ }^{23}$ F. Lüpke, M. Eschbach, T. Heider, M. Lanius, P. Schüffelgen, D. Rosenbach, N. von den Driesch, V. Cherepanov, G. Mussler, L. Plucinski, D. Grützmacher, C. M. Schneider, and B. Voigtländer, "Electrical resistance of individual defects at a topological insulator surface," Nat. Commun. 8, 15704 (2017).

${ }^{24}$ C. Durand, X.-G. Zhang, S. M. Hus, C. Ma, M. A. McGuire, Y. Xu, H. Cao, I. Miotkowski, Y. P. Chen, and A.-P. Li, "Differentiation of surface and bulk conductivities in topological insulators via four-probe spectroscopy," Nano Lett. 16, 2213 (2016).

${ }^{25}$ S. M. Hus, X.-G. Zhang, G. D. Nguyen, W. Ko, A. P. Baddorf, Y. P. Chen, and A. P. Li, "Detection of the spin-chemical potential in topological insulators using spin-polarized four-probe STM," Phys. Rev. Lett. 119, 137202 (2017).

${ }^{26}$ J. Yang, D. Sordes, M. Kolmer, D. Martrou, and C. Joachim, "Imaging, single atom contact and single atom manipulations at low temperature using the new ScientaOmicron LT-UHV-4 STM," Eur. Phys. J.: Appl. Phys. 73, 10702 (2016).

${ }^{27}$ G. Rapenne and C. Joachim, “The first nanocar race,” Nat. Rev. Mater. 2 , 17040 (2017)

${ }^{28}$ R. Ma, Q. Huan, L. Wu, J. Yan, W. Guo, Y.-Y.Zhang, S. Wang, L. Bao, Y. Liu, S. Du, S. T. Pantelides, and H.-J. Gao, "Direct four-probe measurement of grain-boundary resistivity and mobility in millimeter-sized graphene," Nano Lett. 17, 5291 (2017).

${ }^{29}$ K. Li, C. Zhang, Y. Wu, W. Lin, X. Zheng, Y. Zhou, S. Lu, and J. Kang, Nano Lett. 18, 1724 (2018).

${ }^{30}$ See http://www.fz-juelich.de/conferences/4pp_workshop2018 for information about the workshop on multi-tip STM.

${ }^{31} \mathrm{P}$. Hofmann and J. W. Wells, "Surface sensitive conductance measurements," J. Phys.: Condens. Matter 21, 013003 (2009).

${ }^{32}$ T. Nakayama, O. Kubo, Y. Shingaya, S. Higuchi, T. Hasegawa, C.-S. Jiang, T. Okuda, Y. Kuwahara, K. Takami, and M. Aono, "Development and application of multipleprobe scanning probe microscopes," Adv. Mater. 24, 1675 (2012).

${ }^{33}$ A.-P. Li, K. W. Clark, X.-G. Zhang, and A. P. Baddorf, "Electron transport at the nanometerscale spatially revealed by fourprobe scanning tunneling microscopy," Adv. Funct. Mater. 23, 2509 (2013).

${ }^{34} \mathrm{~T}$. $\mathrm{Xu}$ and B. Grandidier, "Electrical characterization of semiconductor nanowires by scanning-probe microscopy," in Semiconductor Nanowires: Materials, Synthesis, Characterization and Applications, edited by J. Arbiol and Q. Xiong (Elsevier, 2015), pp. 277-304, ISBN: 978-1-78242-253-2.

${ }^{35}$ I. Miccoli, F. Edler, H. Pfnr, and C. Tegenkamp, "The 100th anniversary of the four-point probe technique: The role of probe geometries in isotropic and anisotropic systems," J. Phys.: Condens. Matter 27, 223201 (2015).

${ }^{36}$ T. Nakayama, O. Kubo, Y. Shingaya, S. Higuchi, T. Hasegawa, C.-S. Jiang, T. Okuda, Y. Kuwahara, K. Takami, and M. Aono, "Multiple-probe scanning probe microscopes for nanoarchitectonic materials science," Jpn. J. Appl. Phys., Part 1 55, 1102A7 (2016).

${ }^{37}$ B. Voigtländer, V. Cherepanov, and P. Coenen, "The multimeter at the nanoscale," Vak. Forsch. Prax. 28, 38 (2016).

${ }^{38}$ I. Shiraki, F. Tanabe, R. Hobara, T. Nagao, and S. Hasegawa, "Independently driven four-tip probes for conductivity measurements in ultrahigh vacuum," Surf. Sci. 493, 633 (2001).

${ }^{39}$ H. Grube, B. C. Harrison, J. Jia, and J. J. Boland, "Stability, resolution, and tiptip imaging by a dual-probe scanning tunneling microscope," Rev. Sci. Instrum. 72, 4388 (2001).

${ }^{40}$ K. Takami, M. Akai-Kasaya, A. Saito, M. Aono, and Y. Kuwahara, "Construction of independently driven double-tip scanning tunneling microscope,” Jpn. J. Appl. Phys., Part 2 44, L120 (2005).

${ }^{41}$ O. Guise, H. Marbach, and J. T. Yates, Jr., "Development and performance of the nanoworkbench: A four tip STM for conductivity measurements down to submicrometer scales," Rev. Sci. Instrum. 76, 045107 (2005).

${ }^{42}$ W. Yi, I. I. Kaya, I. B. Altfeder, I. Appelbaum, D. M. Chen, and V. Narayanamurti, "Dual-probe scanning tunneling microscope for study of nanoscale metal-semiconductor interfaces," Rev. Sci. Instrum. 76, 063711 (2005).

${ }^{43}$ J. F. Xu, P. M. Thibado, and Z. Ding, "4K, ultrahigh vacuum scanning tunneling microscope having two orthogonal tips with tunnel junctions as close as a few nanometers," Rev. Sci. Instrum. 77, 093703 (2006). 
${ }^{44}$ R. Hobara, N. Nagamura, S. Hasegawa, I. Matsuda, Y. Yamamoto, Y. Miyatake, and T. Nagamura, "Variable-temperature independently driven four-tip scanning tunneling microscope," Rev. Sci. Instrum. 78, 053705 (2007).

${ }^{45}$ V. Cherepanov, E. Zubkov, H. Junker, S. Korte, M. Blab, P. Coenen, and B. Voigtländer, "Ultra compact multitip scanning tunneling microscope with a diameter of 50 mm," Rev. Sci. Instrum. 83, 033707 (2012).

${ }^{46}$ M. Salomons, B. V. C. Martins, J. Zikovsky, and R. A. Wolkow, "Fourprobe measurements with a three-probe scanning tunneling microscope," Rev. Sci. Instrum. 85, 045126 (2014).

${ }^{47}$ See www.scientaomicron.com for information about commercial multi-tip STMs.

${ }^{48}$ See www.rhk-tech.com for information about commercial multi-tip STMs

${ }^{49}$ See www.unisoku.com for information about commercial multi-tip STMs.

${ }^{50}$ See www.mprobes.com for information about commercial multi-tip STMs.

${ }^{51}$ T.-H. Kim, Z. Wang, J. F. Wendelken, H. H. Weitering, W. Li, and A.-P. $\mathrm{Li}$, "A cryogenic Quadraprobe scanning tunneling microscope system with fabrication capability for nanotransport research," Rev. Sci. Instrum. 78, 123701 (2007).

${ }^{52}$ R. Ma, Q. Huan, L. Wu, J. Yan, Q. Zou, A. Wang, C. Bobisch, L. Bao, and H. J. Gao, "Upgrade of a commercial four-probe scanning tunneling microscopy system," Rev. Sci. Instrum. 88, 063704 (2017).

${ }^{53}$ D. K. Schroder, Semiconductor Material and Device Characterization (Wiley-IEEE Press, 2015), ISBN: 978-0-471-73906-7.

${ }^{54}$ F. Lüpke, D. Cuma, S. Korte, V. Cherepanov, and B. Voigtländer, "Fourpoint probe measurements using current probes with voltage feedback to measure electric potentials," J. Phys.: Condens. Matter 30, 054004 (2018).

${ }^{55}$ L. B. Valdes, "Resistivity measurements on germanium for transistors," Proc. IRE 42, 420 (1954)

${ }^{56}$ J. W. Wells, J. F. Kallehauge, and Ph. Hofmann, "Surface-sensitive conductance measurements on clean and stepped semiconductor surfaces: Numerical simulations of four point probe measurements," Surf. Sci. 602, 1742 (2008).

${ }^{57}$ S. Just, H. Soltner, S. Korte, V. Cherepanov, and B. Voigtländer, "Surface conductivity of $\mathrm{Si}(100)$ and $\mathrm{Ge}(100)$ surfaces determined from four-point transport measurements using an analytical N-layer conductance model," Phys. Rev. B 95, 075310 (2017).

${ }^{58}$ F. Krok, M. R. Kaspers, A. M. Bernhart, M. Nikiel, B. R. Jany, P. Indyka, M. Wojtaszek, R. Mller, and C. A. Bobisch, "Probing the electronic transport on the reconstructed $\mathrm{Au} / \mathrm{Ge}(001)$ surface," Beilstein J. Nanotechnol. 5, 1463 (2014).

${ }^{59}$ A. Many, Y. Goldstein, and N. B. Grover, Semiconductor Surfaces (NorthHolland Publishing Co., Amsterdam, 1965).

${ }^{60} \mathrm{H}$. Lüth, Solid Surfaces, Interfaces and Thin Films (Springer, 2015).

${ }^{61}$ P. A. Schumann and E. E. Gardner, "Application of multilayer potential distribution to spreading resistance correction factors," J. Electrochem. Soc. 116, 87 (1969).

${ }^{62} \mathrm{~J}$. Lis, "Electric currents at semiconductor surfaces from the perspective of drift-diffusion equations," Phys. Rev. B 95, 235423 (2017).

${ }^{63} \mathrm{~V}$. M. Tatarnikov, "Use of probes for measuring the electrical conductance of anisotropic plates," Meas. Tech. 13, 877 (1970).

${ }^{64}$ P. Jaschinsky, J. Wensorra, M. I. Lepsa, J. Myslivecek, and B. Voigtländer, "Nanoscale charge transport measurements using a double-tip scanning tunneling microscope," J. Appl. Phys. 104, 094307 (2008).

${ }^{65}$ J. P. Ibe, P. P. Bey, Jr., S. L. Brandow, R. A. Brizzolara, N. A. Burnham, D. P. DiLella, K. P. Lee, C. R. K. Marrian, and R. J. Colton, "On the electrochemical etching of tips for scanning tunneling microscopy," J. Vac. Sci. Technol., A 8, 3570 (1990).

${ }^{66} \mathrm{~S}$. Yoshimoto, Y. Murata, R. Hobara, I. Matsuda, M. Kishida, H. Konishi, T. Ikuno, D. Maeda, T. Yasuda, S. Honda, H. Okado, K. Oura, M. Katayama, and S. Hasegawa, "Electrical characterization of metalcoated carbon nanotube tips," Jpn. J. Appl. Phys., Part 2 44, L1563 (2005).

${ }^{67}$ O. Kubo, Y. Shingaya, M. Nakaya, M. Aono, and T. Nakayama, "Epitaxially grown $\mathrm{WO}_{x}$ nanorod probes for sub-100 nm multiple-scanning-probe measurement," Surf. Sci. 88, 254101 (2006).

${ }^{68}$ S. Yoshimoto, Y. Murata, K. Kubo, K. Tomita, K. Motoyoshi, T. Kimura, H. Okino, R. Hobara, I. Matsuda, S. Honda, M. Katayama, and S. Hasegawa, "Four-point probe resistance measurements using PtIr-coated carbon nanotube tips," Nano Lett. 7, 956 (2007).

${ }^{69}$ B. Voigtländer, Scanning Probe Microscopy: Atomic Force Microscopy and Scanning Tunneling Microscopy (Springer, New York, Berlin, Heidelberg, 2015), ISBN: 978-3-662-45240-0.
${ }^{70} \mathrm{R}$. Hobara, S. Yoshimoto, S. Hasegawa, and K. Sakamoto, "Dynamic electrochemical-etching technique for tungsten tips suitable for multitip scanning tunneling microscopes," e-J. Surf. Sci. Nanotechnol. 5, 94 (2007).

${ }^{71}$ W. D. Pilkey, Formulas for Stress, Strain, and Structural Matrices (John Wiley \& Sons, 2008), ISBN: 9780471032212.

${ }^{72}$ I. Morawski and B. Voigtländer, "Combined frequency modulated atomic force microscopy and scanning tunneling microscopy detection for multitip scanning probe microscopy applications," Rev. Sci. Instrum. 81, 033703 (2010).

${ }^{73}$ I. Morawski, R. Spiegelberg, S. Korte, and B. Voigtländer, "Combined frequency modulated atomic force microscopy and scanning tunneling microscopy detection for multi-tip scanning probe microscopy applications," Rev. Sci. Instrum. 86, 123703 (2015).

${ }^{74}$ P. Jaschinsky, P. Coenen, G. Pirug, and B. Voigtländer, "Design and performance of a beetle-type double-tip scanning tunneling microscope," Rev. Sci. Instrum. 77, 093701 (2006).

${ }^{75} \mathrm{See}$ http://www.fz-juelich.de/pgi/pgi-3/microscope for a movie showing a scanning STM tip imaged by an SEM.

${ }^{76}$ The sample was kindly provided by Grace Lu (NAMI group Nanoelectronics and Advanced Materials Innovations at the University of Southern California, Los Angeles).

${ }^{77}$ Due to the following argument an infinite capacitance can be assigned to two conductors in contact. An infinite amount of charge can be supplied without any voltage developing and due to $C=Q / V$ the capacitance can be assigned as infinite.

${ }^{78}$ F. Lüpke, S. Just, M. Eschbach, T. Heider, E. Mlynczak, M. Lanius, P. Schüffelgen, D. Rosenbach, N. von den Driesch, V. Cherepanov, G. Mussler, L. Plucinski, D. Grützmacher, C. M. Schneider, F. S. Tautz, and B. Voigtländer, "In situ disentangling surface state transport channels of a topological insulator thin film by gating," npj Quantum Mater. 3, 46 (2018).

${ }^{79}$ L. J. van der Pauw, "A method of measuring specific resistivity and Hall effect of discs of arbitrary shape," Philips Res. Rep. 13, 1 (1958).

${ }^{80}$ L. J. van der Pauw, "A method of measuring the resistivity and Hall coefficient on lamellae of arbitrary shape," Philips Tech. Rev. 20, 220 (1958).

${ }^{81}$ R. Rymaszewski, "Relationship between the correction factor of the fourpoint probe value and the selection of potential and current electrodes," J. Phys. E: Sci. Instrum. 2, 170 (1969).

${ }^{82} \mathrm{G}$. Mussler and M. Lanius, private communication (2018).

${ }^{83}$ P. Muralt and D. W. Pohl, "Scanning tunneling potentiometry," Appl. Phys. Lett. 48, 514 (1986).

${ }^{84}$ R. M. Feenstra and B. G. Briner, "The search for residual resistivity dipoles by scanning tunneling potentiometry," Superlattices Microstruct. 23, 699 (1998).

${ }^{85}$ M. Rozler and M. R. Beasley, "Design and performance of a practical variable-temperature scanning tunneling potentiometry system," Rev. Sci. Instrum. 79, 073904 (2008).

${ }^{86}$ T. Druga, M. Wenderoth, J. Homoth, M. A. Schneider, and R. G. Ulbrich, "A versatile high resolution scanning tunneling potentiometry implementation,” Rev. Sci. Instrum. 81, 083704 (2010).

${ }^{87}$ A. Bannani, C. A. Bobisch, and R. Möller, "Local potentiometry using a multiprobe scanning tunneling microscope,” Rev. Sci. Instrum. 79, 083704 (2008).

${ }^{88}$ F. Lüpke, S. Korte, V. Cherepanov, and B. Voigtländer, "Scanning tunneling potentiometry implemented into a multi-tip setup by software," Rev. Sci. Instrum. 86, 123701 (2015)

${ }^{89}$ T. Nakamura, R. Yoshino, R. Hobara, S. Hasegawa, and T. Hirah, "Development of a convenient in situ UHV scanning tunneling potentiometry system using a tip holder equipped with current-injection wires," e-J. Surf. Sci. Nanotechnol. 14, 216 (2016).

${ }^{90}$ R. S. Wagner and W. C. Ellis, "Vapor-liquid-solid mechanism of single crystal growth," Appl. Phys. Lett. 4, 89 (1964).

${ }^{91}$ S. Korte, M. Steidl, W. Prost, V. Cherepanov, B. Voigtländer, W. Zhao, P. Kleinschmidt, and Th. Hannappel, "Resistance and dopant profiling along freestanding GaAs nanowires," Appl. Phys. Lett. 103, 143104 (2013).

${ }^{92}$ A. Nägelein, L. Liborius, M. Steidl, C. Blumberg, P. Kleinschmidt, A. Poloczek, and T. Hannappel, "Comparative analysis on resistance profiling along tapered semiconductor nanowires: Multi-tip technique versus transmission line method," J. Phys.: Condens. Matter 29, 394007 (2017).

${ }^{93}$ C. Gutsche, I. Regolin, K. Blekker, A. Lysov, W. Prost, and F. J. Tegude, "Controllable p-type doping of GaAs nanowires during vapor-liquid-solid growth,” J. Appl. Phys. 105, 024305 (2009). 
${ }^{94}$ A. Nägelein, M. Steidl, S. Korte, B. Voigtländer, W. Prost, P. Kleinschmidt, and Th. Hannappel, "Investigation of charge carrier depletion in freestanding nanowires by a multi-probe scanning tunneling microscope," Nano Res. (published online, 2018).

${ }^{95} \mathrm{See}$ http://www.fz-juelich.de/pgi/pgi-3/mstm for a movie of four-point resistance measurements along a nanowire.

${ }^{96}$ R. M. Feenstra, "A prospective: Quantitative scanning tunneling spectroscopy of semiconductor surfaces," Surf. Sci. 603, 2841 (2009).
${ }^{97}$ G. Münnich, A. Donarini, M. Wenderoth, and J. Repp, "Fixing the energy scale in scanning tunneling microscopy on semiconductor surfaces," Phys. Rev. Lett. 111, 216802 (2013).

${ }^{98}$ W. Melitz, J. Shen, A. C. Kummel, and S. Lee, "Kelvin probe force microscopy and its application," Surf. Sci. Rep. 66, 1 (2011).

${ }^{99}$ C. Saunus, J. R. Bindel, M. Pratzer, and M. Morgenstern, "Versatile scanning tunneling microscopy with 120 ps time resolution," Appl. Phys. Lett. 102, 051601 (2013). 